\title{
Mapping vesicle shapes into the phase diagram: A comparison of experiment and theory.
}

\author{
H.-G. Döbereiner*, E. Evans ${ }^{\dagger}$, M. Kraus ${ }^{*}$, U. Seifert*, and M. Wortis ${ }^{\ddagger}$ \\ *Max-Planck-Institut für Kolloid- und Grenzflächenforschung, \\ Kantstrasse 55, 14513 Teltow-Seehof, Germany \\ ${ }^{\dagger}$ Department of Physics, University of British Columbia, \\ 6224 Agriculture Road, Vancouver, British Columbia, Canada V6T 2A6 \\ ${ }_{\ddagger}^{\ddagger}$ Physics Department, Simon Fraser University, \\ Burnaby, British Columbia, Canada V5A $1 S 6$
}

\begin{abstract}
Phase-contrast microscopy is used to monitor the shapes of micron-scale fluid-phase phospholipid-bilayer vesicles in aqueous solution. At fixed temperature, each vesicle undergoes thermal shape fluctuations. We are able experimentally to characterize the thermal shape ensemble by digitizing the vesicle outline in real time and storing the time-sequence of images. Analysis of this ensemble using the area-difference-elasticity (ADE) model of vesicle shapes allows us to associate (map) each time-sequence to a point in the zerotemperature (shape) phase diagram. Changing the laboratory temperature modifies the control parameters (area, volume, etc.) of each vesicle, so it sweeps out a trajectory across the theoretical phase diagram. It is a nontrivial test of the ADE model to check that these trajectories remain confined to regions of the phase diagram where the corresponding shapes are locally stable. In particular, we study the thermal trajectories of three prolate vesicles which, upon heating, experienced a mechanical instability leading to budding. We verify that the position of the observed instability and the geometry of the budded shape are in reasonable accord with the theoretical predictions. The inability of previous experiments to detect the "hidden" control parameters (relaxed area difference and spontaneous curvature) make this the first direct quantitative confrontation between vesicle-shape theory and experiment.
\end{abstract}

submitted to PRE

Typeset using REVTEX 


\section{INTRODUCTION}

Micron-scale fluid-phase lipid-bilayer vesicles have been observed in recent years under controlled laboratory conditions [1] 11] to exhibit many amusing and diverse shapes. At the same time, there is now a one-parameter theory of vesicle shapes, the so-called areadifference-elasticity (ADE) model [12 15], which appears to be qualitatively consistent with available experimental observations. It would be nice, however, to have a vesicle with an accurately measured shape and known parameters, to plug these parameters into the theory, to predict a shape, and to compare with the measured one. Up to this time, this has not been possible, and, indeed, there have been few (if any) direct quantitative confrontations between theory and experiment.

The reasons for this unsatisfactory state of affairs have their origins in both theory and experiment. On the theoretical side, the principal models which have been proposed to describe vesicle shapes [12,16,18] all have in common the same catalogue of stationary-energy shapes. Thus, simple observation of a vesicle whose shape can be found in the catalogue, while evidence for the general validity of bending-energy models, does not distinguish one variant from another. In order to test the model, it is necessary to study more indirect and/or delicate issues such as stability (absolute and relative) or shape-change systematics under variation of control parameters. This has not often been done [19] for fundamental experimental reasons: First, there are two important vesicle parameters which can be modified systematically in the lab but are not subject to direct measurement. One of these is the spontaneous curvature, $C_{0}$, which measures the preferred radius of curvature of the relaxed bilayer, based on the different lipid composition of the two constituent monolayer leaves and/or the different aqueous environments inside and outside the vesicle. This parameter is presumably the same for all vesicles in a single homogeneous suspension. The other is the relaxed area difference, $\Delta A_{0}$, between the two leaves, based on the different number of lipid molecules which they contain [20] and the long relaxation time for lipid exchange between them [21,22]. This parameter will in general vary from one vesicle to another in the same suspension, based on the (unknown) manner in which vesicle closure occured during preparation and on any interleaf "flip-flop" or intercalation events which may have occured subsequently. In addition, the vesicle shape, itself, is a significantly ambiguous quantity. At laboratory temperatures, all nonspherical vesicles undergo significant and unavoidable thermal fluctuations. Thus, at any nonzero temperature $T$, experiment must characterize a thermal shape ensemble. A single "snapshot," such as has often been exhibited in the previous literature, cannot do this. Finally, experiments have not in practice probed the full, three-dimensional vesicle shape but at best a two-dimensional cut through it at the focal plane of the observing apparatus.

It is the aim of this paper to show how to deal with all these problems in a serious manner for the first time. Using video phase contrast microscopy, we recorded for each vesicle and at each temperature long time-sequences of two-dimensional vesicle contours. We parameterized these images in terms of a set of shape amplitudes. We used the shapeamplitude time-sequences to construct a thermal ensemble, from which we extracted a set of thermal expectation values. Using this data, we show below how to associate each vesicle with a particular point in the phase diagram. In principle, information is left over after the mapping, so that a nontrivial confrontation between theory and experiment is possible. 
In practice, available resolution limits what we can do; nevertheless, nontrivial checks are possible.

Overall, the results are encouraging. The vesicles that we have located in ("mapped into") the theoretical phase diagram do, generally, end up in regions where they are predicted to be locally stable and to have low energies. Furthermore, observed thermal trajectories exhibit shape instability close to (if not always exactly at) positions predicted by the theory. Finally, after the instability the shape is in reasonable agreement with theoretical expectations.

Gravitational effects play an important role experimentally. In order to record long timesequences of vesicle shapes, it is convenient to adjust the density of the exterior solution so that the vesicles have a small negative buoyancy and collect at the floor of the experimental cell, where they remain within the focal plane of the microscope for long periods. In addition, gravity orients the long axis of vesicles of prolate shape so that it stays in or near the focal plane. These are practical issues. On the conceptual side, whenever it has non-neutral buoyancy, a vesicle is subject to gravitational shape deformations. The importance of these deformations has only recently been recognized [23] and was not considered in the analysis of earlier experiments. In this paper, we first perform the full analysis without including gravity. Then, we devote a separate section to the consideration of gravitational corrections. The upshot is that gravitational effects can be significant; however, in the region of the phase diagram upon which we focus attention, there is no change in the qualitatively good agreement between theory and experiment.

The layout of the paper is as follows: Section II introduces the theoretical background necessary to analyse the experiments. Section IIII describes the experimental procedures. Section [V explains how the analysis of the experimental shape contours was carried out. Section $\nabla$ sets forth our results using a pure ADE mapping and ignoring gravitational effects. Finally, in Section VI, we explore the effects of gravity. Section VII provides a final assessment and summary.

\section{BACKGROUND}

\section{A. The area-difference-elasticity model}

In order to have a language for discussing the experiments, it will be useful to present here a summary of some principal features of the ADE model. Additional material is available elsewhere 12 15, 18, 24, 25. At mesoscopic length scales, larger than molecular sizes but smaller than the persistence length, the shape $S$ of a fluid-bilayer vesicle is controlled by an energy functional $W[S]$ consisting of two parts. The first, due to Helfrich [26], measures the overall bending energy and is scaled by the bending modulus $\kappa$. The second requires a brief explanation: Assuming fixed bilayer separation, $D$, the actual area difference between the two leaves of the bilayer is

$$
\Delta A[S]=2 D \oint d A H(\mathbf{r})
$$

where $H(\mathbf{r})$ is the local mean curvature at the point $\mathbf{r}$ of the vesicle surface and the integral

runs over the (closed) vesicle surface. On the other hand, the preferred or relaxed area 
difference,

$$
\Delta A_{0}=\left(N_{\text {out }}-N_{\text {in }}\right) a_{0}(T)
$$

of the two leaves is determined by the difference $\left(N_{\text {out }}-N_{\text {in }}\right)$ between the number of lipid molecules in the outer and inner leaves. The relaxed area $a_{0}(T)$ per lipid molecule is a material parameter but can depend, of course, on the temperature $T$. Once the vesicle has closed, $\Delta A_{0}$ can only change due to lipid flip-flop between the two leaves and/or lipid interchange with the aqueous environment of the vesicle, processes which are believed to be slow on the timescale of the mechanical shape changes we shall be discussing [21,22]. The second contribution to $W[S]$ measures the elastic energy necessary to force $\Delta A[S]$ to differ from $\Delta A_{0}$, when the vesicle assumes the shape $S$. Because the vesicle is fluid, this (local) elastic strain is distributed uniformly over the vesicle surface and appears as an apparently nonlocal term controlled by a so-called nonlocal bending modulus $\bar{\kappa}$. The moduli $\kappa$ and $\bar{\kappa}$ are both of order $K D^{2}$, where $K$ is the area stretching modulus of the bilayer [12], so the ratio,

$$
\alpha \equiv \bar{\kappa} / \kappa
$$

is generically of order unity. The material parameters $\kappa$ and $\bar{\kappa}$ can be measured directly. For SOPC, it is believed that $\kappa \sim 0.90 \pm 0.06 \times 10^{-19} \mathrm{~J}$ [27]. It has been estimated that $\alpha \sim 1.4$ [12]. (A somewhat higher value, $\kappa \sim 1.20 \pm 0.17 \times 10^{-19} \mathrm{~J}$ and a comparable but quite uncertain value of $\alpha$ have been recently observed in tether-pulling experiments [28].) The energy scale $\kappa$ is much smaller than the energies necessary to change significantly the area $A$ and volume $V$ of the vesicle [29], so these quantities may be regarded as fixed in comparing the energies $W[S]$ of different shapes.

Combining the two terms described in the previous paragraph (and dropping an irrelevant, shape-independent term) leads to,

$$
W[S]=\kappa\left[G[S]+\frac{\alpha}{2}\left(m[S]-\bar{m}_{0}\right)^{2}\right],
$$

where

$$
G[S]=\frac{1}{2} \oint d A(2 H)^{2}
$$

which is the starting point of our theoretical discussion of shapes. In writing Eq. (四), we have chosen to rescale all lengths in terms of an "area length" $R_{A}$ defined by $A \equiv 4 \pi R_{A}^{2}$. Thus, the area difference appears in the reduced form,

$$
m[S]=\Delta A / 2 D R_{A}
$$

and the relaxed area difference combines with the spontaneous curvature $C_{0}$ into a single effective reduced area difference,

$$
\bar{m}_{0}=m_{0}+2 c_{0} / \alpha,
$$

where $m_{0}=\Delta A_{0} / 2 D R_{A}$ and $c_{0} \equiv C_{0} R_{A}$ is the reduced value of the spontaneous curvature. Because $C_{0}$ and $\Delta A_{0}$ appear only in the combination $\bar{m}_{0}$, it is impossible in principle to 
detect either one separately by a single shape measurement. Note that the bracketed terms in Eq. (4) are all dimensionless ratios of lengths, invariant under a scale change of the shape $S$, provided that at the same time $C_{0}$ is changed to keep $c_{0}$ fixed. In this sense, $W[S]$ depends on the shape of $S$ but not its overall size. An appropriate scale-independent volume measure is the reduced volume $v \equiv 3 V / 4 \pi R_{A}^{3}$, which lies in the interval $[0,1]$.

To be a mechanically viable shape for a vesicle with given $A$ and $V, S$ must make the energy (4) stationary at the corresponding values of $v$ and $\bar{m}_{0}$ [30], i.e., it must satisfy

$$
\delta W=0=\kappa\left(\delta G[S]-\alpha\left(\bar{m}_{0}-m[S]\right) \delta m[S]\right) .
$$

In general, there are several distinct branches of stationary shapes, which we label $S^{(n)}\left(v, \bar{m}_{0}\right)$, with corresponding energies $W^{(n)}\left(v, \bar{m}_{0}\right)$. To be a candidate for observation in the lab, a shape $S^{(n)}\left(v, \bar{m}_{0}\right)$ must, in addition, be locally stable to small shape perturbations [31]. The lowest-energy branch (which must, of course, be stable) defines the ground state and should, in principle, be observed at sufficiently long times when the temperature is low. However, when energy barriers are large on the scale of $k_{B} T$, other low-lying locally stable branches may remain metastable for long periods. For SOPC, $\kappa \sim 20 k_{B} T_{\text {room }}$ [27], so metastability is expected to be common.

Finally, we shall need below an important connection between the ADE-model shapes and those of the so-called spontaneous curvature (SC) model [26.32], defined by the energy functional,

$$
W_{S C}[S]=\frac{\kappa}{2} \oint d A\left(2 H-\bar{C}_{0}\right)^{2}=\kappa\left(G[S]-2 \overline{c_{0}} m[S]+\text { const. }\right),
$$

which describes a model without differential area elasticity and having a spontaneous curvature $\bar{C}_{0}\left(\bar{c}_{0}\right.$ is the corresponding reduced spontaneous curvature). The variation of Eq. (9) gives a condition which has the same form as Eq. (8) only with the replacement,

$$
2 \bar{c}_{0} \equiv \alpha\left(\bar{m}_{0}-m\left[S^{(n)}\right]\right) .
$$

It follows that any stationary shape $S^{(n)}$ of the ADE model (4i) is also a stationary shape of a spontaneous curvature model (9) with the $\bar{c}_{0}$ defined by Eq. (10), which we shall henceforth refer to as the effective reduced spontaneous curvature for the ADE shape $S^{(n)}$. Notice that the control parameters enter the variational shape equation (8) entirely via the coefficient of the second term. It follows that the stationary shape $S^{(n)}$ is specified completely and in a way that is independent of $\alpha$ by giving $v$ and $\bar{c}_{0}$. For this reason, it will sometimes be convenient in what follows to think of the stationary shapes as $S^{(n)}\left(v, \bar{c}_{0}\right)$ rather than as $S^{(n)}\left(v, \bar{m}_{0}\right)$, which still depends implicitly on $\alpha$. The variation (8) may be thought of as proceeding in two steps: First make $G[S]$ stationary at fixed $m$, thus defining a function $G^{(n)}(v, m)$, then subsequently carry out the variation with respect to $m$. It follows from Eq. (8) that $\bar{c}_{0}$ can be evaluated as

$$
2 \bar{c}_{0}=\frac{\partial G^{(n)}(v, m)}{\partial m}=2 c_{0}+\alpha\left(m_{0}-m\left[S^{(n)}\right]\right) .
$$




\section{B. $T=0$ Phase diagram and the stability of prolate shapes}

The map in the $\left(v, \bar{m}_{0}\right)$ plane of the regions where various branches provide the lowest energy shape constitutes the $T=0$ (shape) phase diagram for the ADE model. This phase diagram, which depends on $\alpha$, can now be constructed rather easily numerically, at least, for $v$ not too small and when $\bar{m}_{0} / 4 \pi$ is not too far from unity [12, 33, 34]. The experiments described in this paper deal with a branch of axisymmetric shapes, called "prolates," because they have up/down symmetry and resemble prolate ellipses when they have reduced volume not too much below unity. The region of the ADE phase diagram in which these prolates appear is shown in Fig. 1. It is bounded below by oblate axisymmetric shapes and by a region of non-axisymmetric shapes, which need not concern us further here [35. Above the prolates lies a region of pear shapes, for which axisymmetry remains but the up/down symmetry has been broken. This pear region is, in turn, bounded above by a line $L^{\text {pear }}$ of fully "vesiculated" limiting shapes, consisting of two spheres joined by a narrow neck. The region above $L^{\text {pear }}$ is incompletely explored but believed to be dominated by additional interconnected vesiculated shapes. The boundary between the prolates and pears at relatively high reduced volume involves a discontinuous shape change (corresponding to a simple crossing of the energy branches $W^{\text {pear }}$ and $W^{\text {pro }}$ ) along the line $D^{\text {pro/pear }}$ but a continuous shape change (corresponding to a bifurcation of $W^{\text {pear }}$ away from $W^{\text {pro }}$ ) along the line labeled $C^{\text {pro/pear }}$, for lower reduced volumes beyond the tricritical point $T$. Both transitions are often called "budding."

It is important to emphasize that the prolate shape branch continues to exist outside of the "prolate region" of the phase diagram. Indeed, within the context of the ADE model and in the region of reduced volume shown in Fig. 1, a stationary prolate shape exists for every value of $\bar{m}_{0}$ [36]. These shapes can only be observed, of course, when they are locally stable. The region of local prolate stability includes the "prolate region" of the phase diagram but extends beyond it into metastable regions, where the true ground state has some other shape. It is a crucial test of the theory that prolate shapes observed in the lab should, indeed, map to the region of predicted prolate (local) stability.

Metastability boundaries are marked by the first appearance of a soft mode, i.e., a family of fluctuations which lower the overall energy. The region of Fig. 11 within which prolates are predicted by the ADE model to be locally stable is bounded above by the line $M_{0,-}^{\text {pro }}$ and below by the line $M_{2,+}^{\text {pro }}$. These lines are calculated by an analysis of constrained Gaussian fluctuations about the calculated stationary shape [34]. The subscripts label the rotation mode $|m|$ and (even/odd) parity of the sector where the first instability occurs. It is an important result of this theory [37] that instabilities in sectors which break the symmetry are a property of the shape $S^{(n)}$ alone, while those in non-symmetry-breaking sectors depend in addition independently on $\alpha$. For the prolate shapes, the boundaries $C^{\text {pro/pear }}$ and $M_{0,-}^{\text {pro }}$ in Fig. 1 both reflect instability in the symmetry-breaking sector $(|m|=0$, odd parity ). These boundaries are, thus, independent of $\alpha$ in a $\left(v, \bar{c}_{0}\right)$ representation of the phase diagram, so, in the usual $\left(v, \bar{m}_{0}\right)$ representation, they shift with $\alpha$ according to

$$
\bar{m}_{0}=m\left[S^{\text {pro }}\left(v, \bar{c}_{0}\right)\right]+\frac{2 \bar{c}_{0}}{\alpha} .
$$

These lines of shape instability (and not the actual shape (phase) boundaries!) are the 
experimentally relevant (observable) ones.

Strictly speaking, the above picture holds only in the low-temperature limit, since for $T>0$ sharp phase boundaries do not exist, because the vesicle is a finite system with finite energy and always explores its full phase space. Nevertheless, in practice, as long as the prolate branch remains locally stable and surrounded by energy barriers appreciably larger than $k_{B} T$, thermally fluctuating prolate shapes are readily seen in the lab. In the prolate region of the phase diagram, these fluctuating shapes constitute a true, stationary, equilibrium ensemble. In regions which are only metastable, the set of prolate shapes should be regarded as a restricted ensemble, which may, however, be quasi-stationary for appreciable periods of time. In practice, metastability is expected to break down slightly inside the boundaries $M^{\text {pro }}$, when the metastability barrier becomes comparable to $k_{B} T$.

\section{Prolate shapes for reduced volumes near unity: The hierarchy and the mapping}

We review briefly here what is known theoretically about the $T=0$ stationary shapes $S^{\text {pro }}\left(v, \bar{c}_{0}\right)$ for the relatively high reduced volumes which will be relevant for the experiments 12,34.

Prolate shapes are axisymmetric. Therefore, they are completely described by the curve made by their intersection with any plane which includes the symmetry axis. This curve may be written in terms of an arclength $s$ which starts at the north pole $(s=0)$ and ends at the south pole $\left(s=s^{*}\right)$. We take the direction of the polar axis to be $\hat{y}$ and the perpendicular direction to be $\hat{x}$. A representation which will be convenient for our purposes is

$$
\psi(s)=\pi \frac{s}{s^{*}}+\sum_{n=1}^{\infty} a_{n}^{(0)} \sin \left(n \pi \frac{s}{s^{*}}\right)
$$

where $\psi(s)$ is the angle between $\hat{y}$ and the outward-pointing normal to the curve. The overall length scale is set by $s^{*}$. Note that $\psi(0)=0$ and $\psi\left(s^{*}\right)=\pi$. The first term on the right describes a semicircular arc, i.e., a spherical vesicle shape. The coefficients $\left\{a_{n}^{(0)}\right\}$ parameterize deviations from the sphere. For shapes like prolates, which are up-down symmetric, the odd- $n$ coefficients vanish. We note as an aside that the coefficients $\left\{a_{n}^{(0)}\right\}$ cannot be set independently, since closure of the curve at the south pole requires that

$$
x\left(s^{*}\right)=\int_{0}^{s^{*}} d s \cos \psi(s)=0 .
$$

This places a complicated nonlinear condition on the set $\left\{a_{n}^{(0)}\right\}$, which for any real vesicle shape will automatically be satisfied.

The stationary shapes of the prolate branch are given by the coefficients $a_{2 n}^{(0)}\left(v, \bar{c}_{0}\right)$. It is clear that, when $v$ is near unity, the coefficients $a_{2 n}^{(0)}$ will all be small. It is a consequence of the stationarity condition (8) that these non-vanishing coefficients have the structure of a well-defined hierarchy 25],

$$
\begin{array}{rrr}
a_{2}^{(0)}=A_{2}(1-v)^{\frac{1}{2}}+B_{2}\left(\bar{c}_{0}\right)(1-v)+O\left((1-v)^{\frac{3}{2}}\right), & +B_{4}\left(\bar{c}_{0}\right)(1-v)+O\left((1-v)^{\frac{3}{2}}\right), \\
a_{4}^{(0)}= & O\left((1-v)^{\frac{3}{2}}\right), \\
a_{6}^{(0)}= &
\end{array}
$$


where $A_{2}=(135 / 64)^{1 / 2} \simeq 1.45$ and the coefficients $B_{2}$ and $B_{4}$ are linear functions of $\bar{c}_{0}$ with coefficients of order unity. These results follow from Refs. [12] and [24]. It is a consequence of this structure that for $v$ near unity $a_{2}^{(0)} \gg a_{4}^{(0)} \gg a_{6}^{(0)} \ldots$, a hierarchy which reflects the fact that modes of higher $n$ correspond to shorter wavelengths and cost more bending energy. $a_{2}^{(0)}$ is independent of $\bar{c}_{0}$ at lowest order, because this is the only contribution of order $(1-v)^{\frac{1}{2}}$ and is, therefore, entirely determined by the constraint on the reduced volume $v$.

Figure 2, which was calculated by solving numerically the variational equation (8), illustrates the dependence of $a_{4}^{(0)}$ on $v$ and $\bar{c}_{0}$ for the prolate branch near $v=1$. It is clear that for $v<0.95$ the terms of order $(1-v)^{\frac{3}{2}}$ and higher have an appreciable effect. Note that, except near the anomalous point [38] $v \simeq 0.85$, knowledge of $a_{4}^{(0)}$ and $v$ uniquely determines $\bar{c}_{0}$.

This brings us, finally, to the issue of the "mapping", i.e., of associating an experimentally observed $T=0$ prolate vesicle shape with a point in the ADE phase diagram, Fig. 1. Knowing the vesicle shape means that we have direct experimental access to "geometrical" quantities such as $v$ and $m$, through the shape coefficients $a_{2 n}^{(0)}$. The abscissa, $v$, of the phase diagram is geometrical; however, the ordinate, $\bar{m}_{0}$, Eq. (元), encodes information about the initial area difference $\Delta A_{0}$ and the spontaneous curvature $C_{0}$, which are neither geometric nor directly observable in any other way. The solution to this apparent impasse is to use $a_{4}$ and Fig. 2 to infer a value of $\bar{c}_{0}\left(v, a_{4}^{(0)}\right)$, the effective reduced spontaneous curvature, which is not observable, and to combine this with the then (theoretically) fixed $m\left(v, \bar{c}_{0}\right)$ to

calculate $\bar{m}_{0}$ (Eq. (10) ). Note that, in principle, any of the nonzero coefficients $\left\{a_{2 n}^{(0)}\right\}$ could be used to produce such a mapping, $\bar{c}_{0}\left(v, a_{2 n}^{(0)}\right)$. In practice, however, as the hierarchy (15) shows, $a_{2}^{(0)}$ is very insensitive to $\bar{c}_{0}$ (because $A_{2}$ is independent of $\bar{c}_{0}$ and $B_{2}$ is only weakly dependent on it) and $a_{6}^{(0)}$ is sufficiently small so that experimental noise makes it a poor candidate.

This framework is still incomplete in three senses. First, what the experiment observes is not a single $T=0$ shape but an ensemble of thermally fluctuating shapes. Second, the mapping as described above simply takes an experimental point and associates it in a one-toone manner with a point in the theoretical phase diagram. It does not yet in any obvious way test the correspondence between theory and experiment. Third, effects of gravity should be taken into account. We discuss these important points in Secs. IV, Q, and V1, respectively. But, before doing so, we turn to the experiments.

\section{EXPERIMENTAL TECHNIQUES}

\section{A. Materials and Preparation}

For all experiments, vesicles were prepared from the common phospholipids 1Stearoyl-2-Oleoyl-sn-Glycero-3-Phosphatidylcholine (SOPC) or 1,2-Dimyristoyl-sn-Glycero3-Phosphatidylcholine (DMPC). These lipids have their main phase transitions at $5^{\circ} \mathrm{C}$ and $23^{\circ} \mathrm{C}$, respectively [39]. They were purchased in powder form (Avanti Polar Lipids, Birmingham, AL, USA) and stored dissolved in chloroform:methanol (2:1) in special chemically 
inert glass vials (Fisher Scientific) below $-15^{\circ} \mathrm{C}$.

Preparation was done using standard techniques [25,40,41]: A few drops $(30 \mu \mathrm{l})$ of lipid solution (10 mg per ml chloroform:methanol) are spread with a syringe needle on a roughened Teflon disk. The solvent is evaporated in a vacuum chamber overnight. The disk with the dried lipid is placed in a glass beaker $(50 \mathrm{ml})$ and pre-hydrated with a stream of Argon saturated with water vapor for about 20 minutes. Then, the desired solution for vesicle swelling is added and the beaker is covered with Parafilm and placed in the oven. To avoid heat shock, the solution and the beaker with the Teflon disk are heated separately to the swelling temperature prior to incubation. Swelling was done with $50 \mathrm{mMol}$ sucrose solution at a temperature of $36^{\circ} \mathrm{C}$.

Successful vesicle development is indicated by whitish streaks in the swelling solution. These streaks are collected into an Eppendorf tube with cleaned glass pipettes and incubated at the swelling temperature. Excess glucose solution $(48 \mathrm{mMol})$ is then added to obtain the desired density for the vesicles in the observation chamber. The end result of this procedure is a vesicle suspension with an interior sucrose solution and an exterior glucose solution (with a slight admixture of sucrose). The excess density of the interior relative to the exterior sugar solution is approximately $3.3 \mathrm{~g} / \mathrm{l}$. This is needed in order that the vesicles sink gently to the bottom of the experimental cell, as discussed further below. Vesicles were stored at the swelling temperature and used within a few days.

\section{B. Experimental Setup and Data Acquisition}

For observation, vesicles are placed in a specially designed microchamber, tightly sealed with glass plates above and below to prevent evaporation [25]. Temperature is monitored by a thermocouple inserted into the observation chamber. A water bath, incorporated integrally into the chamber, provides temperature uniformity and control at the level of $\pm 0.1^{\circ} \mathrm{C}$. Because their density is slightly higher than that of the surrounding solution, the suspended vesicles fall to the bottom of the cell, where they rest gently against the lower plate and are observed from below via video phase-contrast microscopy. We use a standard inverted Leitz microscope equipped with phase contrast (Leitz Phaco 40/0.65), capable of an overall magnification of 500 times and incorporating annular illumination and a phase ring. The light source was a $\mathrm{Hg}$ arclamp powered by a high-voltage transformer. A permanent green filter and various grey filters were used to minimize degradation of the lipids. The video camera was positioned above the eye piece in such a way as to gain a resolution of 86 nm per pixel in a $480 \times 480$ frame.

The visible phospholipid structures [42] which collect at the bottom of the observation chamber in the microscope's focal plane are typically very diverse [4, ,7], including topologically complex and multilamellar structures, small vesicles included within larger ones, vesicles connected to one another by sub-microscopic tethers or tubes, vesicles with obvious adhesions, etc. For detailed observation, we try to select simple, topologically spherical, unilamellar structures, without identifiable microscopic connections or adhesions. In addition, we monitor the fluctuations of each candidate vesicle for some time prior to data acquisition in order to reject those with obviously "abnormal" behavior, e.g., those exhibiting unexplainable asymmetry or sudden changes in apparent area or volume. The final fraction of 
usable vesicles is less than one percent.

Images of selected vesicles are simultaneously displayed on the video monitor, saved to tape (U-Matic, Sony), and processed in real time, as will be described in the next subsection. To sample at a fixed temperature a single thermal "shape ensemble" takes about $20 \mathrm{~min}$. Recording a thermal shape trajectory requires data at several different temperatures for the same vesicle. The chamber is allowed to equilibrate for at least $5 \mathrm{~min}$. after each temperature change. Temperature is recorded with a precision of $\pm 0.1^{\circ} \mathrm{C}$. The total amount of data gathered consists of over 80 hours of video tape of more than 150 vesicles, including a wide range of shapes. Budding is an ubiquitous process, which we observed at least 15 times in a controlled fashion. It is important to get long runs at each fixed temperature in order properly to sample the full thermal shape ensemble. On the other hand, thermal-trajectory runs which extend over more than a few hours appear to be contaminated by systematic drifts, presumably due to lipid degradation and/or flip-flop between bilayer leaves, which establishes intrinsic limits on sampling density and run time.

In this paper we restrict analysis to three particular SOPC vesicles (A, B, and C), which have in common that they started with prolate elliptical shapes and, on heating, eventually underwent budding transitions, as illustrated in Figs. 3-6. Qualitatively, other vesicles monitored behaved similarly, although they followed different trajectories, some exhibiting sharp shape transitions and others not. The reason for selecting the budding trajectories is that the location of the budding instability provides a particularly stringent test of the theory, as we shall discuss in Sec. $\nabla$.

\section{Processing the Video Image}

In order to analyse the data, it is necessary to reduce the video image to a time sequence of digitized shape contours. This was done by using a frame grabber (Matrox, Dorval, Quebec, Canada) to capture each image, computer processing the image in real time, storing the digitized contour point in memory, and then grabbing a new image. The image processing algorithm (described below) requires between 0.4 and $0.6 \mathrm{~s}$ (depending on vesicle size) on a PC with a 486 DX CPU and a $66 \mathrm{MHz}$ clock speed. Thus, for a video frequency of 30 frames/s, we are processing every 15th frame. This is relatively slow compared to processing times on the order of $0.1 \mathrm{~s}$, which have been reported in the literature [5,43]. However, in contrast to these fast procedures, our algorithm has a better-than-pixel accuracy in finding the contour 44,45]. This high resolution turns out to be critical to the success of our experiments, since we shall need to resolve small changes in mean shape in tracking the thermal trajectory 46.

In phase-contrast microscopy the image of the vesicle edge exhibits a "halo," with a light band (intensity maximum) just outside the vesicle and a dark band (intensity minimum) just inside. Typically, the intensity profile crosses the gray value of the local background at its steepest point, and we have taken this point to be the nominal position of the vesicle boundary 47.

The contour-digitizing algorithm is fully described in Ref. [25]. The algorithm works on the (integer) pixel grid $\left(n_{x}, n_{y}\right)$ and requires initialization by hand to the vicinity of the outline of the particular vesicle to be studied (there are ordinarily several vesicles in the 
field of view). Suppose that a scan in $n_{x}$ at fixed $n_{y}=n_{y}^{(0)}$ intersects the halo. By averaging the grey values in the vicinity of the halo, we establish a local background intensity. The profile of intensity-versus- $n_{x}$ crosses this background value at a point $x$ (generally noninteger) which may be determined by linear interpolation. The point $\left(x, n_{y}^{(0)}\right)$ is then stored as a contour point, and the algorithm steps $n_{y} \rightarrow n_{y}+1$ and starts again. Note that the interpolation procedure allows determination of the $x$ coordinate of the contour point with better-than-pixel precision. Whenever the contour profile becomes steeper when scanned in the $y$ direction rather than in the $x$ direction, the algorithm automatically switches to scanning $n_{y}$ at fixed $n_{x}$, and vice versa. Each contour is terminated at closure. Motion of the vesicle between successive frames is normally small enough so that each subsequent frame can be started where the earlier one terminated, so the initialization step needs to be carried out only at the beginning of each run 48.

The digitized contours exhibit noise at the pixel level ( 1 pixel $=86 \mathrm{~nm})$. This behavior presumably reflects the intrinsic noise of the original optical signal, the pixelation statistics, the digitization of the grey scale, and other factors. To remove some of this microscopic noise before data analysis, it is convenient to smooth the observed contours. This was done by applying a tenth-order binomial filter [49] to the $x$ and $y$ contour coordinates, thus averaging over an effective width of about 5 pixels. The distribution of deviations of the original data points from the smoothed contour is Gaussian with a typical full width at half maximum of about 0.7 pixels, thus giving an effective local lateral resolution of about $30 \mathrm{~nm}$ (compared to a typical vesicle size of several microns). This resolution, well below the nominal optical resolution given by the wavelength of light, illustrates the delicate line-shape discrimination achievable via phase contrast [50] and is more than adequate for quantifying the overall vesicle shape and the low-lying fluctuation modes.

The result of this process is a time-sequence of several thousand digitized contours, illustrating the shape ensemble of each vesicle at each temperature. The relationship of these two-dimensional contours to the three-dimensional vesicle shape requires a brief discussion. The "general wisdom" seems to be that what is seen in phase contrast microscopy is a cut through the vesicle in the focal plane [5, 43, 51 [54]. However, this is an oversimplification. Phase contrast is particularly sensitive to edges, so vesicle boundaries which "overhang" the focal plane (relative to the optical axis) may contribute to the image to a greater or lesser extent depending on the focal depth and the amplitude of the edge contrast. Following the practice of the literature, we shall ignore such effects in what follows. We wish only to point out that there are substantive issues here which deserve to be addressed more fully in future work.

In collecting data, the microscope is focussed on the maximal cross section of the vesicle under observation, and this focal plane does not change over time. For vesicles such as $\mathrm{A}, \mathrm{B}$, and $\mathrm{C}$ which are (on the average) prolate and axisymmetric, gravity tends to orient the symmetry axis horizontally, i.e., to bring it into the focal plane. Thus, the contours (such as those based on Figs. 3- 6), which constitute our raw data, may be thought of as an ensemble of cuts through the mean symmetry axis of the fluctuating vesicle. Fluctuations of the symmetry axis out of the horizontal plane modify this simple picture: If the focal plane no longer includes the symmetry axis, then the depth of focus and the edge enhancement mentioned in the previous paragraph probably give the resulting image the character of a 
projected outline of the tilted vesicle. In principle, this projected shape is different from a true axial section. In practice, the stabilizing effect of gravity is large enough so that these out-of-plane fluctuation effects are almost always small (except near the spinodal line of the budding transition), so we will treat the two-dimensional contours as if they represent axial sections. Note that there is a balance here. In order to keep the theoretical analysis simple, we would like to ignore the effects of gravity on the vesicle shape. On the other hand, in order to perform the experiment conveniently, we use gravity to localize the vesicle in the bottom of the chamber and to orient the symmetry axis (of prolate vesicles) to the horizontal plane (see further discussion at the end of Sec. V).

\section{ANALYSIS OF THE TWO-DIMENSIONAL SHAPE CONTOURS}

This Section describes how we parameterize the individual digitized two-dimensional shape contours discussed in Sec. [II], how we average the shape parameters over each thermal ensemble, and how we infer $T=0$ three-dimensional shape information from these averaged parameters.

\section{A. Thermal fluctuations of the vesicle shape: General discussion}

Varying the temperature has two effects. On the one hand, it modifies, through ordinary thermal expansion, the temperature-dependent control parameters, $A(T), V(T)$, and $\Delta A_{0}(T)$, as well as the material control parameters, $\kappa, \bar{\kappa}$, and $C_{0}$, which appear in the Hamiltonian (4). These effects produce the so-called "thermal trajectories," which we shall discuss in Sec. $\nabla$. On the other hand, even if all these parameters were temperature independent, there would still be ordinary thermal fluctuations. It is for the moment these purely thermal fluctuations to which we direct our attention.

There is, in principle, no way of taking a single fluctuating shape contour and inferring the corresponding $T=0$ shape. At best, we must take a full thermal shape ensemble and use theory to infer the $T=0$ shape of the vesicle with the same control parameters. When the fluctuations are large, even this is beyond present theoretical capability. However, when fluctuations are small enough so that they may be treated at the Gaussian level, progress can be made.

The upshot of a recent study of Gaussian fluctuations of vesicles of arbitrary axisymmetric shapes [37, 34,55] may be summarized as follows: Any typical fluctuating shape may be regarded as a $T=0$ shape appropriately translated and rotated (the so-called Euclidean modes) plus an area- and volume-conserving normal (i.e., perpendicular) displacement $u(\mathbf{r})$ at each point $\mathbf{r}$ of the surface. It is a special feature of these fluctuations that (because of the strict area and volume constraints) both the average displacement $\langle u(\mathbf{r})\rangle$ and the mean-square fluctuations $\left\langle u^{2}(\mathbf{r})\right\rangle$ are generically of the order $k_{B} T / \omega$, where $\omega$ is a typical static fluctuation-mode energy. Note that the rms fluctuations are always larger than the shift when the fluctuations are small. In the analysis which follows, we shall assume that the Gaussian regime holds and we shall ignore the mean shifts. Ordinarily, the fluctuation-mode energies $\omega$ are of the order $\kappa$. For our vesicles $\kappa / k_{B} T \simeq 20$, so for most regions of the phase diagram, this is an excellent approximation. There is, however, an important exception. At 
the instability boundaries (Fig. 1) one of the modes becomes soft. Thus, near enough such boundaries, the Gaussian treatment fails, and we may expect difficulties (see Sec. \).

\section{B. Parameterization of the two-dimensional shape contours}

We interpret the measured two-dimensional contours as being sections which include the principal symmetry axis of the vesicle (Sec. III $)$. Thus, in the spirit of the last paragraph and up to corrections which are normally of order $k_{B} T / \kappa$, the center of mass is located in (i.e., near) the focal plane at (i.e., near) the point which is the center of mass of the digitized contour. We determine this point numerically for each contour. We then find the (approximate) principal (long) vesicle axis by calculating the two-dimensional moment-ofinertia tensor with respect to the center of mass and diagonalizing. This determines the (nominal) principal axis of each vesicle shape and identifies the north and south poles. We call the direction of the principal axis $\hat{y}$ and the corresponding perpendicular direction $\hat{x}$ (which is, of course, not necessarily a principal axis of the three dimensional vesicle). Thus, each experimental contour is reduced to a set of points $\left\{x_{i}, y_{i}\right\}$. In what follows, we treat each half-contour separately (each image has two half contours) and take $x_{i} \geq 0$.

It is convenient to represent each half-contour in the angle-arclength $\psi(s)$ representation of Sec. IIC by calculating

$$
\psi_{i}=-\arctan \left(\frac{y_{i+1}-y_{i-1}}{x_{i+1}-x_{i-1}}\right),
$$

where the arctangent is defined on its Riemann surface, i.e., $\psi(s)$ is continuous at the equator. The arclength $s$ is measured from the north pole. The parameterization parallels Eq. (13),

$$
\psi(s)=\pi \frac{s}{s^{*}}+\sum_{n=1}^{\infty} a_{n} \sin \left(n \pi \frac{s}{s^{*}}\right) .
$$

The coefficients $\left\{a_{n}\right\}$ are obtained by a numerical integration using the trapezoidal rule,

$$
a_{n}=(-1)^{n} \frac{2}{n}+\sum_{i=1}^{M}\left(\psi_{i} \sin \left(n \pi \frac{s_{i}}{s^{*}}\right)+\psi_{i+1} \sin \left(n \pi \frac{s_{i+1}}{s^{*}}\right)\right) \frac{s_{i+1}-s_{i}}{s^{*}}
$$

and, henceforth, they replace the points $\left\{x_{i}, y_{i}\right\}$ in representing the half-contour. ( $M$ is the number of digitized points in the half-contour.) Note that the contours here are not up-down symmetric, so the odd-n coefficients do not in general vanish, as they did for the $T=0$ prolate shapes. Similarly, $\psi(0)$ and $\psi\left(s^{*}\right)$ are normally nonzero.

\section{Thermal ensembles and $T=0$ shapes}

For each half-contour of each video image, we calculate the shape coefficients $\left\{a_{n}\right\}$ plus the nominal ("effective") vesicle area and volume,

$$
A_{e}=\pi \sum_{i=1}^{M}\left(x_{i}+x_{i+1}\right)\left(s_{i+1}-s_{i}\right)
$$


and

$$
V_{e}=-\frac{\pi}{2} \sum_{i=1}^{M}\left(x_{i}^{2}+x_{i+1}^{2}\right)\left(y_{i+1}-y_{i}\right) .
$$

In a similar spirit, we compute an effective reduced volume,

$$
v_{e}=\frac{V_{e}}{\frac{4 \pi}{3}\left(\frac{A_{e}}{4 \pi}\right)^{\frac{3}{2}}} .
$$

For axisymmetric vesicle shapes, these equations would calculate the true area, volume, and reduced volume, respectively. Since each image is only a snapshot of a section through a fluctuating vesicle, $v_{e}$ is only approximately equal to the true reduced volume $v$. Note that $v_{e}$ fluctuates in time for successive images of a given vesicle at a fixed temperature, while $v$ is in principle constant, since the true area and volume are conserved during the shape fluctuation.

The several thousand images which constitute a typical experimental run with a given vesicle at a fixed temperature lead to characteristic time series for the quantities $\left\{a_{n}\right\}$ and $v_{e}$, as illustrated by Figs. 7 and 8 . Although the series are noisy, we expect to see memory effects between successive images, as long as there are any characteristic physical relaxation times longer than the $0.5 \mathrm{~s}$ between successive grabbed images. We have estimated elsewhere [10] the typical relaxation times expected for these vesicles. Away from instabilities, the characteristic times are expected to be at most several seconds, which is consistent with direct visual observations of the optical image. As the vesicle approaches an instability (which occured at $v=0.878$ for vesicle $\mathrm{A}$ ), one sees rapidly increasing relaxation times, corresponding to a spinodal slowing-down [10]. This tendency is clearly visible when comparing Figs. 7 and 8. As long as the data set spans a time interval much larger than the longest relaxation time, we may expect that the time sequence samples an effective stationary ensemble [56]. In this sense, the thermal ensemble of fluctuating shapes is characterized by the set of ensemble averages $\left\langle a_{n}\right\rangle,\left\langle a_{m} a_{n}\right\rangle,\left\langle v_{e}\right\rangle,\left\langle v_{e}^{2}\right\rangle$, etc. Indeed, the distribution functions $P\left(a_{n}\right), P\left(v_{e}\right)$, etc., are typically Gaussian form in shape [25].

In order to proceed with the mapping, we now need a procedure for inferring the zerotemperature quantities $v$ and $\left\{a_{n}^{(0)}\right\}$ from the thermal data. We do this in the crudest way, by simply making the identifications,

$$
v=\left\langle v_{e}\right\rangle \text { and } a_{n}^{(0)}=\left\langle a_{n}\right\rangle .
$$

As explained above (Sec.IVA), the justification for these identifications is that the averaging process suppresses the rms fluctuations, which are of order $\sqrt{k_{B} T / \omega}$. This leaves the thermal shifts plus the terms of order $\left\langle u^{2}\right\rangle$, both of which scale as $k_{B} T / \omega$, which we ignore in first approximation [57]. These values of $v$ and $a_{4}^{(0)}$ allow us (Sec. IIC) to infer $\bar{c}_{0}$ (and, thereby, $\bar{m}_{0}$ ) from Fig. 2, and, thus, to complete the mapping.

It is hard to give any meaningful estimate of the real uncertainty in the derived quantities $v$ and $a_{n}^{(0)}$. For a truly stationary ensemble, the purely statistical (sampling) uncertainties in the average quantities should decrease as the ensemble sampling becomes denser. In practice, our runs are necessarily of finite length (Sec. IIIB). Indeed, if we divide the data 
set into two parts, corresponding to earlier and later times, we typically see a spread of values corresponding typically to \pm 0.001 for $v$ and \pm 0.002 for the $\left\{a_{n}^{(0)}\right\}$ 's (and somewhat larger near the budding instability). It is this measure which we adopt as an estimate of the statistical uncertainties. Of course, there are also systematic errors, such as the thermal shifts (which we have neglected), the failure of the Gaussian picture (where fluctuations are large), the sampling error (when the relaxation times are long), the fluctuations of the major axis out of the focal plane, and the effects of gravity (Sec. VI). The upshot is that well away from the instability boundary the statistical uncertainties are probably realistic, except for the systematic influence of gravitational effects. Near the instabilities, the situation is less well defined. These statistical uncertainties translate (via Fig. 2) into uncertainties in $\bar{c}_{0}$, as we shall illustrate in Sec. V.

\section{RESULTS (WITHOUT GRAVITATIONAL CORRECTIONS)}

Each of the three budding vesicles, A,B, and C, started at a relatively low temperature with a nearly spherical shape (i.e., $v \approx 1$ ). As the temperature was raised, the reduced volume decreased, until at a certain temperature (different for the different vesicles) a "budding" instability occurred (see Figs. 5 and 6), i.e., the vesicle suddenly necked down and, over a time interval of $1-10$ seconds, developed a small quasispherical satellite. (This time range is due to the different vesicle sizes, since typical relaxation times scale with the third power of the vesicle radius [10].) Up to the budding threshold, the thermally induced changes in the fluctuating ensemble are reversible to within experimental precision. The budding process, itself, is a mechanical instability [10]. In fact, the budding can be reversed, but only by cooling to a temperature significantly below the budding temperature [58. The size of the fluctuations and the scale of the longest relaxation time increases dramatically as the temperature approaches the budding temperature (see Figs. 3, 4, 0, and 8). These effects have been interpreted in terms of a simple Landau theory [10].

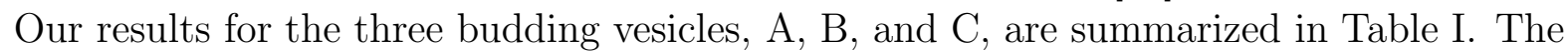
average amplitudes $\left\langle a_{n}\right\rangle$ were generally very small for odd $n$, as expected in the prolate phase [59]. The even coefficients $\left\langle a_{6}\right\rangle$ and above were too small to distinguish from zero, presumably because of the hierarchy (15). The value $v_{b}$ of the reduced volume at budding was determined by extrapolating the experimental temperature dependence $v(T)$ to the observed budding temperature $T_{b}$.

Figure 9 shows the result of mapping this data into the theoretical $\left(v, \bar{c}_{0}\right)$ diagram by using $\left\langle v_{e}\right\rangle,\left\langle a_{4}\right\rangle$, and Fig. 2, as explained in Sec. IIC. The instability lines $M^{\text {pear }}$ and $M^{\text {obl }}$ are just the appropriately mapped versions of the corresponding spinodal lines of Fig. 11. The advantage of this representation is that it is completely independent of the value of $\alpha$, as explained after Eq. (10). Theory predicts that the prolate shapes are locally stable only between the two spinodals. With the exception of the highest-temperature point in the trajectory of vesicle A, we see that the mapped shapes do lie in this region. Fig. 10 shows the same data plotted in the $\left(v, \bar{m}_{0}\right)$ phase diagram, Fig. 11. The required relation between $\bar{c}_{0}$ and $\bar{m}_{0}$ is based on Eq. (10). In order to evaluate $m\left[S^{(n)}\right]$, we solve the variational shape equations derived from energy functional Eq. (9) for the given values of $v$ and $\overline{c_{0}}$. We have taken $\alpha=1.4$ in making this transformation [28,32]. Since the spinodal boundaries map 
right along with the data points, there is no change in the predicted stability.

The fact that, with a single exception, the mapped data points lie neatly sandwiched in the region of predicted (local) stability is a stringent quantitative test of the theory and constitutes the single most important result of this work. We emphasize again (see Sec. IIB) that the shapes mapped by Fig. 2 are variationally stationary (by construction) but not necessarily locally stable, so that an arbitrary shape could end up anywhere in the phase diagram.

It is worth pointing out that fact that the values of $\bar{c}_{0}$ derived from the data points are all of order unity (as expected on the basis of the theory) is also an important test. Fig. 2 shows that $\bar{c}_{0}$ values between -5 and 10 are associated with values of $a_{4}^{(0)}$ in the narrow range between -0.02 and 0.02 . If the theory were significantly in error, it would be quite easy to have produced very large or very small values of $\bar{c}_{0}$.

Indeed, in a certain sense, our ability reliably to distinguish shape changes corresponding to differences of order unity in $\bar{c}_{0}$ is, in itself, surprising. Consider that, for a vesicle of radius $10 \mu \mathrm{m}$ at a reduced volume $v=0.9$, a difference in $\bar{c}_{0}$ of \pm 1 corresponds to a change in shape which modifies the pole-to-pole contour length $s^{*}$ by only $20 \mathrm{~nm}$. This number (the smallness of which is a direct consequence of the hierarchy) is below the local lateral resolution of the contour. How is this possible? First, one has to realize that one does not measure a single distance only. Rather, the amplitudes are calculated globally from an integral (see Eq. (18)) over about 600 contour points, each of which deviates from the reference shape. Second, one is interested in a low mode, which is insensitive to local perturbations in the membrane. And, third, the amplitudes are averaged over typically several thousand contours, giving an effective sample size on the order of $10^{5}$. Thus, shape differences on the $10 \mathrm{~nm}$ scale are detectable [50].

The "thermal trajectories" corresponding to each vesicle encode the effect of the experimental control parameter (temperature) on the quantities $v$ and $\bar{c}_{0}$ (or $\bar{m}_{0}$ ), defined in Sec. IIA. These quantities, in turn, depend on the volume $V$, area $A$, relaxed area difference $\Delta A_{0}$ of the vesicle, on the thickness $D$ and spontaneous curvature $C_{0}$ of the membrane, and on the ratio $\alpha$ of elastic constants. All these quantities are in principle temperature dependent, and, if these dependences were known, we could calculate the thermal trajectory and compare with that found in Figs. 9 and 10. The volume thermal expansion coefficient ( $\beta_{V} \approx 3 \times 10^{-4} / \mathrm{K}$ for water) is known to be small compared to the area thermal expansion coefficient $\left(\beta_{A} \approx 3 \times 10^{-3} / \mathrm{K}\right.$ for $\mathrm{SOPC}$ 60]). It is also known [61] that the total bilayer volume $A D$ is only weakly temperature dependent. A simple model is to assume that only $A$ and $D$ are temperature dependent. When $C_{0}=0$, as is reasonable for a symmetric bilayer, this assumption leads to the simple result that the product $v \bar{m}_{0}$ is temperature independent [16,25]. This hypothesis predicts trajectories of the general shape and scale shown in Figs. 9 and 10 but significantly less steep than those observed. It is not hard to make more refined models consistent with the data, for example, by using a non-zero spontaneous curvature $C_{0}$ and/or a differential thermal expansion for the two leaves of the bilayer [16,25]. Unfortunately, direct measurements of these quantities are not available, so no useful conclusions can be drawn at this stage.

Another set of evidence bearing on the consistency of the observations with the theory is the relative size and shape of the main vesicle and the bud which forms at the instability. 
At the crudest level, theory predicts that the final state after budding will be pear shaped (rather than fully vesiculated, with a microscopically narrow neck) when the budding occurs for reduced volumes less than $v_{c}=0.875$, where the spinodal crosses the limiting-pear line $L^{\text {pear }}$ (see Fig. (1). As $\alpha$ increases, this crossing point moves to higher values of $v$, so the observation that vesicle A buds to a fully vesiculated state would be inconsistent with a value of $\alpha$ larger than 1.4. This observation places an upper bound on $\alpha$. Another qualitative prediction is that the ratio $r$ of the bud radius to the radius of the remaining main vesicle should increase as the reduced volume at budding decreases. Thus, $r$ should be largest for vesicle $\mathrm{A}$ and smallest for vesicle $\mathrm{C}$, as is, indeed, observed. On the other hand, on the limiting line $L^{\text {pear }}$, the vesiculated configuration consists of two spheres, so there is a simple relation between the ratio $r$ and reduced volume $v$ [32,62]. In particular, close examination

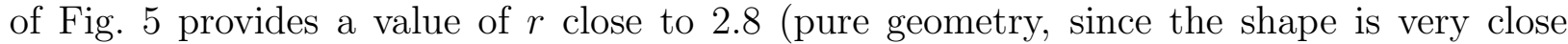
to being two spheres). This corresponds to $v(r)=0.873$. Indeed, budding is observed at $v_{b}=0.878 \pm 0.002$ for vesicle A.

Overall, the agreement of theory and experiment is reasonable with the exception of the last point of the thermal trajectory of vesicle $\mathrm{A}$, which lies distinctly above the theoretical spinodal boundary $M^{\text {pro }}$ (Figs. 9 and 10). This last point is worrying. Indeed, even below the spinodal line, there should be a (fuzzy) unstable region where the energy barrier out of the metastable state is of order $k_{B} T$. (The fact that vesicles $\mathrm{B}$ and $\mathrm{C}$ appear to bud increasingly below $M^{\text {pro }}$ for higher reduced volume suggests that there may be some systematic effect at work which is distorting the locus of instability.) We have considered three possible reasons for this discrepancy.

First, our identification (22) involves the assumptions that (a) the fluctuations are small enough to be treated at the Gaussian level and (b) the (Gaussian) thermal shifts and rms fluctuations (of order $k_{B} T / \omega$ ) can be neglected. At the spinodal, fluctuations diverge [10], so neither of these assumptions is valid, and the identification (22) is expected to fail. It is entirely plausible that these assumptions are already breaking down near the spinodal, at the last stable point. Because the effects of fluctuations beyond the Gaussian level have yet to be calculated, we cannot at this stage assess the impact that such corrections might have on the near-spinodal points of trajectory A

Second, we have assumed that the major prolate axis is (effectively) in the focal plane of the microscope. If this axis is appreciably out of the focal plane, then the digitized images cannot be thought of as sampling axial sections of the three-dimensional fluctuating shape, and the whole analysis of these images would have to be redone. As long as fluctuations are small, it is reasonable to assume that gravity acts to keep the prolate axis aligned. But, near the spinodal line, there are large, slow pear-like fluctuations [10], which are not "updown" symmetric (i.e., which break the symmetry between the north and south poles). In this situation, gravity may be expected to systematically re-orient the small end of the pear towards the bottom of the chamber, thus tipping the effective symmetry axis away from the horizontal. Once tipped, the symmetry axis is inhibited by gravity from returning to the horizontal, so one expects long intervals of asymmetric, pear-like data to appear in the near-spinodal time-sequences. In fact, the data for the last point of the vesicle A trajectory do show an "anomalous" cluster of frames with simultaneously large $a_{3}$ and $a_{4}$, and these frames exhibit a fuzzy contour profile near the small end of the pear, indicating an overhang of the vesicle membrane beyond the focal plane. The effect of excluding this segment of the 
time-sequence is to lower $\left\langle a_{4}\right\rangle$ nearly to the spinodal, thereby improving agreement between theory and experiment.

Finally, we have so far treated the effect of gravity as only something which positions the vesicles at the bottom of the chamber and aligns (prolate) axes in the horizontal plane. In fact, it will also modify the zero-gravity shape analysis which has been up to this point the basis of our mapping procedure. What effect do gravitational shape changes have on the analysis and can they explain the observed discrepancies? This is the subject of the next Section.

\section{GRAVITATIONAL EFFECTS}

\section{A. Qualitative considerations}

When the density of the solution which fills the vesicle interior is greater than that of the exterior solvent, the vesicle will fall to the bottom of the container and, once in contact with the bottom, will deform in such a way as to decrease the gravitational potential energy of the interior, higher-density material. The overall shape involves a balance between the previous bending energy (身) and a new gravitational energy,

$$
W_{\text {grav }}[S]=g_{0} \Delta \rho \int z d V
$$

where $g_{0}$ is the local acceleration due to gravity, $\Delta \rho$ is the excess mass density of the interior solution, $z$ measures height above the bottom of the chamber, and the integral is over the interior volume of the vesicle.

The ratio of the energy scale $g_{0} \Delta \rho R_{A}^{4}$ of this gravitational term to the scale $\kappa$ of the bending energy defines a dimensionless parameter,

$$
g \equiv \frac{g_{0} \Delta \rho R_{A}^{4}}{\kappa}
$$

which measures the relative size of gravitational and bending energies [23]. When $g$ is very small, we may expect shapes which are not significantly deformed relative to the gravity-free case. When $g$ is very large, gravitational energy dominates and vesicles will tend towards circular pancakes [63], squashed against the chamber bottom, insofar as constraints on area and volume allow. (Of course, if $v=1$, then the vesicle can only be spherical.)

Experimental values for our vesicles A, B, and C were nominally $g=2.2,0.3$, and 1.9 , respectively [64]. Here, we use a value of $\kappa=0.9 \times 10^{-19} J$ for our estimation [4]. We may, thus, expect gravitational corrections to be appreciable for vesicles A and $\mathrm{C}$ but relatively less important for vesicle B. The qualitative effect of gravity on the mapping is not hard to see. Roughly speaking, a "pancake" deformation will make the focal-plane section of a prolate rounder and larger in area than it would otherwise be. Therefore, gravitational corrections will lead to larger values of $v_{e}$. Although the coefficients $a_{n}$ must approach zero for large g, it is not obvious where the asymptotic regime sets in, so the sign of the gravitational shift in $a_{4}$ cannot be inferred a priori. In order to estimate these effects quantitatively, we need to be able to calculate vesicle shapes in the presence of gravity. 


\section{B. $T=0$ shapes in the presence of gravity}

The only previous calculation of vesicle shapes including gravitational effects was done by Kraus et al. [23]. These authors found a gravity-induced prolate-oblate transition for values of $g$ similar to those encountered in our experiments. Following this work, we use a polyhedral discretization of the vesicle surface and employ the program Surface Evolver [65] to search iteratively for the shape-energy minimum. Numerical minimization in the presence of a hard-wall constraint for the chamber floor leads to special problems in stability. For this reason, we replaced the hard wall by a soft substrate potential, $V_{\mathrm{w}}(z)=V_{0 \mathrm{w}} \exp \left(-z / z_{0}\right)$, with $V_{0 \mathrm{w}}=5 \kappa$ and $z_{0}=0.1 R_{A}$. These parameters seem to provide a good compromise between numerical stability (favored by a softer potential) and a deformation of the shape caused by the soft tail of the potential which is as small as possible [23. Constraints on area and volume are respected. The energy is minimized by moving the vertices in the direction of the energy gradient or, alternatively, by a conjugate gradient method. Symmetries such as mirror planes can be exploited. For most of this work, only a vertically-cut quarter section of the vesicle was actually computed. It turns out that the results for the final shape and energy are very sensitively dependent on the triangulation in a way that we cannot completely control. We have tried to overcome this problem by fitting a linear interpolation to a grid of data points, as described in Sec. VI B below. This procedure averages out random fluctuations from one point to another but cannot address any subtle systematic dependence on grid size which might be hidden beneath the fluctuations (we did check directly for such a grid-size effect, and none is apparent at the level of accuracy we can achieve). In the absence of a more reliable measure, we have simply used the deviation of the computed data points from the smoothed interpolation to give an estimate of the error introduced by the triangulation.

Finally, we point out that, not only does gravity influence the shape and energy of a vesicle at given $v$ and $\bar{c}_{0}$, but it also changes the relative energy of different shape branches, thus shifting phase boundaries and stability boundaries in the phase diagram. Thus, in looking for gravitational corrections to the experiments, we also need to compute the gravitationally shifted spinodal line $M^{\text {pro }}$. We have done this in the $\left(v, \bar{c}_{0}\right)$ representation (Sec. 【IA) for $g=2.2$, in which now (c.f., Eq.(11))

$$
2 \bar{c}_{0}=\frac{\partial G(v, m)}{\partial m}+\frac{1}{\kappa} \frac{\partial W_{\text {grav }}(v, m)}{\partial m}=2 c_{0}+\alpha\left(m_{0}-m\left[S^{(n)}\right]\right) .
$$

This involves two numerical fits to Surface Evolver data, first for the computation of $m\left(M^{\text {pro }}\right)$ and then for the derivative which evaluates $\bar{c}_{0}$. As a consequence, the quality of the results is rather poor. As shown in Fig. 9, the result for our experiments is a shift of the upper spinodal upward in $\bar{c}_{0}$ by about one unit; but, the numerical uncertainties are unfortunately comparable in size to the shift. More detailed calculations would require finer triangulations and much longer relaxation times. Since the computational investment is already appreciable and experimental uncertainties are already large near the spinodal, additional investment at this time does not seem wise. We did not compute the location of the full lower spinodal including gravity; however, for $\bar{c}_{0}=0$ (and $g=2.2$ ), we do know 23] that the prolate-oblate transition occurs at $v=0.94$ (as plotted), which also corresponds to a small upward shift. 


\section{Gravitational corrections to the mapping}

We estimated gravitational effects by running the Surface Evolver program for $v$ between 0.875 and 0.975 in steps of 0.025 and for $\bar{c}_{0}$ between 2.5 and 10.0 in steps of 2.5. For each pair of these parameters, we computed shapes over a range of small $g$ values. For each shape, we took a maximal horizontal section and computed effective values of $v_{e}, a_{2}$, and $a_{4}$ using formulas (18) and (21). These values varied in a roughly linear way with $g$, only with some superimposed fluctuations which we attributed to the triangulation sensitivity mentioned in the previous subsection. We then assigned effective values of $v_{e}(g), a_{2}(g)$, and $a_{4}(g)$ for the gravitationally distorted shapes by making a straight-line fit to these computed points passing through the values previously computed for $g=0$. For the values of $g, v$, and $\bar{c}_{0}$ relevant to the experiments, the gravitational shift of $a_{4}$ is comparable to the numerical errors. On the other hand, the difference between $v_{e}(g)$ and the actual reduced volume $v$ (see Fig. 11) does lead to a noticeable correction in the values of $\bar{c}_{0}$ inferred from the experimental data for vesicles $\mathrm{A}$ and $\mathrm{C}$.

This family of lines may then be used to calculate gravitational corrections to the experimental data. Since $g$ is known, it is only necessary to take the measured values of $v_{e}$ and $a_{4}$, which belong (presumably) to shapes which are gravitationally distorted, and to infer the corresponding values of $v$ and $\bar{c}_{0}$. Figure 11 shows, for example, the calculated gravitational shift in the apparent volume, $\left(v_{e}-v\right)$, at $g=2.2$ (appropriate for vesicle A) for representative values of $v$ and $\bar{c}_{0}$. Note that the $v_{e}$ is always larger than $v$, in agreement with the qualitative argument of Sec. VI A, so that gravitational correction always shifts the data points to the left in Fig. 9. The corrections increase for values of $\bar{c}_{0}$ close to the prolate-oblate transition, i.e., for small $\bar{c}_{0}$, where increasing the volume of a prolate vesicle at fixed $\bar{c}_{0}$ leads eventually to a transition to an oblate shape with a vertical symmetry axis, thus producing a circular focal-plane section (i.e., $v_{e}=1$ ). The behavior of the shift of the apparent volume near this prolate-oblate transition can be understood as follows: Above the transition, for $v>v_{c}\left(\bar{c}_{0}, g\right)$, the focal cut of the oblate vesicle is circular, independent of $\left(c_{0}, g\right)$, i.e.,

$$
v_{e}-v=1-v, \quad v>v_{c}
$$

For $v<v_{c}$, we find from geometry $v_{e}=1-\frac{64}{135} a_{2}^{2}+O\left(a_{2}^{4}, a_{4}^{2}, \cdots\right)$. Furthermore, the amplitude $a_{2}$ is given by $a_{2} \approx c\left(v_{c}-v\right)^{\frac{1}{2}}$, where the coefficient $c$ depends on both $\bar{c}_{0}$ and $g$. Thus, immediately below the transition, we have

$$
v_{e}-v \simeq 1-\frac{64}{135} c^{2} v_{c}+\left(\frac{64}{135} c^{2}-1\right) v, \quad v \lesssim v_{c} .
$$

This equation implies that all the shifts in the apparent reduced volume for different $\bar{c}_{0}$ meet

the curve $v_{e}-v=1-v$ with a slope, $\left(\frac{64}{135} c^{2}-1\right)$, larger than -1 . Inspection of Fig. 11 suggests that the slope is, in fact, positive for small $\bar{c}_{0}$.

Figures 9 and 10 show the gravitationally corrected (phase) diagrams with the corrected data points for vesicles $\mathrm{A}$ and $\mathrm{C}$. The uncertainties of the gravitationally corrected points include both the original experimental uncertainties and the numerical uncertainties of the gravitational shape-energy calculations. Note that the data points are shifted to the left 
in $v$, as expected qualitatively. All experimental points are in the (metastable) prolate phase, except the "bad" point which still remains above the upper spinodal, unless the "anomalous" cluster is removed, as discussed in the last section. Accepting this somewhat ad hoc procedure, one may argue that this last stable point at $v=0.890$ lies within error bars inside the prolate phase after the gravitational corrections have been performed. We note, however, that the actual point of budding at $v_{b}=0.878$, which must in principle be beneath the spinodal, would still appear to lie slightly in the unstable region, even after gravitational and tipping corrections. We may speculate that this apparent inconsistency is due to thermal shifts (neglected so far in our treatment), which could be appreciable near the spinodal.

So far, we have discussed stability of vesicle $A$ only with respect to the upper (pear-mode) spinodal. We have also checked that the data points of vesicle $A$ fall above the lower limit of stability of the prolates, which is an instability towards the oblate phase. In fact, the first point of vesicle $A$ is located (including the gravity correction) at $\left(v=0.937, \bar{c}_{0}=1.6\right.$ ). The location of the prolate-oblate transition at the same volume (and $g=2.2$ ) is known to occur at $\bar{c}_{0}=0$ (see the previous section), which is comfortably below our data point. Thus, the vesicle-A trajectory does (properly) start in the stable-prolate region. On the other hand, this first point still appears somewhat out of line with the remaining three points of the trajectory (see Fig. 10), which (after gravity corrections) fit quite well to the simple form [12], $\bar{m}_{0} v=$ const. We may speculate that thermal shifts play a role here, too, near the lower spinodal 66.

A few comments are in order concerning gravitational corrections to the vesicle-C data. The reduced volume of vesicle $C$ is shifted to the left, as expected. Unfortunately, the effective spontaneous curvature $\bar{c}_{0}$ is not well determined, due to the large experimental and numerical errors. (We remind the reader that vesicle $C$ did bud at this location and, thus, exhibited large spinodal fluctuations.) This data point appears to be located appreciably below the spinodal line. This could be an artifact created by effects not included in the mapping (see the discussion at the end of Sec. V) and/or it could be due to a low activation energy for budding near the sphere.

The upshot of this exploration of gravitational corrections is that gravity does, indeed, have a substantial numerical effect, as might be anticipated from the fact that the dimensionless parameter $g$ is around 2 for vesicles A and C. However, the qualitative (and generally encouraging) conclusions of the gravity-free analysis are not changed.

\section{CONCLUSION}

Previous experiments (e.g., Ref [6]) have compared experiment with theory by, in effect, exhibiting a set of control parameters $\left(v, c_{0}, m_{0}, \alpha\right)$ which lead to theoretical shapes similar to those observed in the laboratory. It is important that this exercise can be successfully carried through; but, for various reasons, it constitutes far less than a full test of the theory.

The first problem is that different variants of the theory, ranging from the SC model $(\alpha=0)$ to the $\Delta A$ (bilayer-couple) model $(\alpha=\infty)$, all share the same set of stationary shapes, so that observation of a shape which can be suitably parameterized only distinguishes models in which the shape is stable from those in which it is not. One would like to 
be able to measure all the control parameters for a given vesicle and then to verify that a vesicle with those control parameters does, indeed, have the observed shape. The difficulty is that, while $v$ and $\alpha$ are measurable, $c_{0}$ and $m_{0}$ (which enter the shape problem in the combination $\bar{m}_{0}$ ) are not. We have surmounted this problem by concentrating on the equivalent variable $\bar{c}_{0}$ (which incorporates $c_{0}, m_{0}$, and $\alpha$ ) and inferring this variable directly from the shape data $\left(a_{4}\right)$. Although the inference process uses theory, there are nontrivial checks left over. Local stability is still an important check, as we have argued. In particular, the observed (reasonable) agreement of the experimental budding boundary with the calculated theoretical spinodal is encouraging, as is the qualitative agreement of the post-budding shape with that predicted by the theory. Once the mapping is done, observation of other shape coefficients $\left(a_{2}, a_{6}\right.$, etc., for the prolates) provides, in principle, a further test of agreement between theory and experiment. Unfortunately, at the level of precision we have been able to achieve here, $a_{2}$ is too weakly dependent on $\bar{c}_{0}$ to be useful, and $a_{6}$ is too small.

The second and in many ways more important advance which our experiment makes over previous ones is in the monitoring and analysis of the full thermal shape ensemble. Previous workers have certainly observed the shape fluctuations; however, shape comparisons between theory and experiment have heretofore relied on comparison of a single judiciously selected image with a theoretically calculated shape. When fluctuations are appreciable (which they certainly become near any instability boundary), this process is clearly unacceptable. We have illustrated how to monitor and to analyse the full shape ensemble, and we have shown how to relate the ensemble data to the corresponding $T=0$ theoretical shapes, at least in situations where fluctuations are not too large. Treatment of larger fluctuations, which are common near instabilities and will certainly be increasingly important at low $v$ (where mechanical modes will tend to be softer), will require a new theoretical approach capable of going beyond the Gaussian level.

Finally, at a somewhat technical level, we have illustrated that the effects of gravity, which have been ignored in earlier work, are quantitatively important. And, we have shown how to adjust for them in comparing theory and experiment.

In summary, our work provides in principle a quantitative test of the ADE model of vesicle shapes. Agreement between theory and experiment (including suitable corrections) is crude but satisfactory. It is important at this point (and entirely feasible) to carry out similar analyses in other parts of the phase diagram. When more precision becomes available in future experiments, more consistency checks will be possible (e.g., by looking at $\left\langle a_{2}\right\rangle$ and $\left\langle a_{6}\right\rangle$ ), and it will be worthwhile to include in the analysis the corrections of order $k_{B} T / \omega$, which we have ignored herein. It is clear that gravitational corrections will have to be included and that non-Gaussian effects will be important near instabilities.

\section{ACKNOWLEDGMENTS}

We thank M. Jarić, J. Käs, L. Miao, K. Ritchie, E. Sackmann, and T. Yeung for helpful discussions, and R. Lipowsky for generous support. Technical advice and help from A. Leung and W. Rawicz is appreciated. This work was supported in part by the Natural Sciences and Engineering Research Council of Canada (H.-G.D. and M.W.) and by the Medical Research Council of Canada (E.E.). 


\section{REFERENCES}

[1] Useful review articles include, e.g., R. Lipowsky, Nature (London) 349, 475 (1991), and U. Seifert, and R. Lipowsky, in Structure and Dynamics of Membranes, Vol. 1, edited by R. Lipowsky and E. Sackmann (Elsevier, Amsterdam, 1995).

[2] E. Sackmann H. P. Duwe, and H. Engelhardt, Faraday Discuss. Chem Soc. 81, 281 (1986).

[3] E. Sackmann, H. P. Duwe, K. Zeman, and A. Zilker, in Structure and Dynamics of Nucleic Acids, Proteins, and Membranes, edited by E. Clementi and S. Chin (Plenum, New York, 1986), p. 251.

[4] E. Evans and W. Rawicz, Phys. Rev. Lett. 64, 2094 (1990).

[5] H. P. Duwe, J. Käs, and E. Sackmann, J. Phys. (Paris) 51, 945 (1990).

[6] K. Berndl, J. Käs, R. Lipowsky, E. Sackmann, and U. Seifert, Europhys. Lett. 13, 659 (1990).

[7] J. Käs and E. Sackmann, Biophys. J. 60, 825 (1991).

[8] E. Farge and P. Devaux, Biophys. J. 92, 347 (1992).

[9] H.-G. Döbereiner, J. Käs, D. Noppel, I. Sprenger, and E. Sackmann, Biophys. J. 65 1396 (1993).

[10] H.-G. Döbereiner, E. Evans, U. Seifert, and M. Wortis, Phys. Rev. Lett. 75, 3360 (1995).

[11] W. Wintz, H.-G. Döbereiner, and U. Seifert, Europhys. Lett. 33, 403 (1996).

[12] L. Miao, U. Seifert, M. Wortis, and H.-G. Döbereiner, Phys. Rev. E 49, 5389 (1994), which contains additional references.

[13] S. Svetina, A. Ottova-Lietmannová, and R. Glaser, Theor. Biol. 94, 13 (1982).

[14] S. Svetina and B. Žekš, Biomed. Biochim. Acta 42, S86 (1983); 44 , 979 (1985).

[15] S. Svetina and B. Žekš, Eur. Biophys. J. 17, 101 (1989).

[16] U. Seifert, K. Berndl, and R. Lipowsky, Phys. Rev. A 44, 1182 (1991).

[17] B. Fourcade, L. Miao, M. Rao, M. Wortis, and R. K. P. Zia, Phys. Rev. E 49, 5276 (1994).

[18] U. Seifert, Adv. Phys., in press.

[19] The best previous example is probably Ref. [6], where several thermal trajectories were "fit" to the bilayer-couple model. This involved comparing observed and calculated shapes under the assumption of constant integrated mean curvature $m$. The parameters of each trajectory were chosen to fit experimental shapes without any independent confirmation. Single video frames, chosen from what was certainly a fluctuating ensemble, were taken to represent the experimental shapes.

[20] B. L.-S. Mui, H.-G. Döbereiner, T. D. Madden, and P. R. Cullis, Biophys. J. 69, 930 (1995).

[21] R.D. Kornberg and H.M. McConnell, Biochem. 10, 1111 (1971).

[22] R. Homa, and H.J. Pownall, Biochim. Biophys. Acta 938, 155 (1988).

[23] M. Kraus, U. Seifert, and R. Lipowsky, Europhys. Lett. 32 , 431 (1995).

[24] L. Miao, PhD thesis, Simon Fraser University, 1993 (unpublished).

[25] H.-G. Döbereiner, PhD thesis, Simon Fraser University, 1995 (unpublished).

[26] W. Helfrich, Z. Naturforsch. 28c, 693 (1973).

[27] E. Evans and D. Needham, J. Phys. Chem. 91, 4219 (1987). The measurement was at $15^{\circ} \mathrm{C}$. 
[28] R.E. Waugh, J. Song, S. Svetina, and B. Žekš, Biophys. J. 39, 974 (1992).

[29] The stretching modulus $K$ of a phospholipid membrane is roughly $0.1 \mathrm{~J} / \mathrm{m}^{2}$, so the energy necessary for significant change in area of a micron-scale object is about $10^{-13} \mathrm{~J}$. Similarly, the energy necessary to resist significant osmotic pressure is large on the scale of $\kappa$, so the volume of the vesicle is effectively determined by osmotic balance between the inside and the outside solutions.

[30] We regard $\kappa, \alpha, C_{0}$, and $D$ as material parameters, unaffected by any shape changes.

[31] That is, except for the trivial Euclidean modes of translation and rotation, any change in the shape $S$ which keeps $A$ and $V$ constant must cost energy.

[32] L. Miao, B. Fourcade, M. Rao, M. Wortis, and R.K.P. Zia, Phys. Rev. A 43, 6843 (1991).

[33] V. Heinrich, S. Svetina, and B. Žekš, Phys. Rev. E 48, 3112 (1993).

[34] M. Jarić, U. Seifert, W. Wintz, and M. Wortis, Phys. Rev. E 52, 6623 (1995).

[35] These phase boundaries are weakly first order [33,34]. Prolate-oblate shape fluctuations have been observed. See H.-G. Döbereiner and U. Seifert, Europhys. Lett. 36, 325 (1996).

[36] In the spontaneous curvature model, there is a prolate shape for any $\bar{c}_{0}$. As $\bar{c}_{0} \rightarrow \pm \infty$, these shapes reach limiting shapes with finite $m$ [6, 16]. The mapping equation (10) then implies that in the ADE model a stationary prolate exists for any $\bar{m}_{0}$.

[37] U. Seifert, Z. Phys. B 97, 299 (1995).

[38] Figure 2 shows that $a_{4}^{(0)}\left(v=0.85, \bar{c}_{0}\right) \simeq-0.021$ for all values of $\bar{c}_{0}$. We have checked numerically and find that the common crossing point is only approximate. We do not understand the reason for this near degeneracy. What is clear, however, is that in the region near $v=0.85$ measurement of $a_{4}^{(0)}$ for a prolate is not a good predictor of $\bar{c}_{0}$. Our data is at higher reduced volumes, so this is not a problem.

[39] D. Marsh, Handbook of Lipid Bilayers, (CRC Press, Boca Raton, 1990).

[40] D. Needham and E. Evans, Biochem. 27, 8261 (1988).

[41] D. Needham, Methods in Enzymology 220, 111 (1993).

[42] Structures with length scales below $0.2 \mu \mathrm{m}$ are visible only as interference patterns or not at all. Nevertheless, we infer that such structures are present, since we can frequently see their effects - attachments, adhesions, etc. - on the visible structures.

[43] P. Méléard, J. Faucon, M. Mitov, and P. Bothorel, Europhys. Lett. 19, 267 (1992).

[44] A. Dougherty, P.D. Kaplan, and J.P. Gollub, Phys. Rev. Lett. 58, 1652 (1987).

[45] A. Dougherty and J.P. Gollub, Phys. Rev. A 38, 3043 (1988).

[46] This interval of $0.5 \mathrm{~s}$ is too coarse to follow dynamical motion, except for the very slow modes which occur close to the budding instability [10. We could, of course, retrieve the taped video images and achieve a resolution of $30 \mathrm{~Hz}$, but not in real time. However, for present purposes, what we need is only a statistical sample of the full thermal shape ensemble, so following the dynamics is unnecessary. We note that our current set up allows now shape analysis at video frequency.

[47] T. Wilson and C. J. R. Sheppard, Optik 59, 19 (1981), calculated the form of the expected halo for a straight-edged object, assuming a circular phase disk. They find that the position of the edge does, indeed, coincide with the profile-slope maximum. In principle, this calculation should be redone for each specific vesicle shape with the parameters of our optical system. We have not done this calculation in detail but we 
estimate that any deviations from the simple crossing rule will be too small to affect our data appreciably.

[48] Every so often the search algorithm loses the target vesicle between successive frames, e.g., when when another vesicle passes across the field. Such events are identified by monitoring a parameter like the overall contour length. The difficult frame is then discarded and the next available frame is grabbed for analysis.

[49] The coordinates of the contour were averaged 10 times using $x_{i}^{n+1}=\frac{1}{4}\left(x_{i-1}^{n}+2 x_{i}^{n}+x_{i+1}^{n}\right)$, and an analogous expression for $y$.

[50] Note that we do not claim to be able to resolve structures which are only $30 \mathrm{~nm}$ apart. It is only the relative position of the membrane which can be detected with high precision.

[51] M.B. Schneider, J.T. Jenkins, and W.W. Webb, J. Phys. France 45, 1457 (1984).

[52] H. Engelhardt, H. Duwe, and E. Sackmann, J. Phys. Lett. 46, L 395 (1985).

[53] I. Bivas, P. Hanusse, P. Bothorel, J. Lalanne, and O. Aguerre-Chariol, J. Phys. 48, 855 (1987).

[54] J. Faucon, M. Mitov, P. Méléard, I. Bivas, and P. Bothorel, J. Phys. France 50, 2389 (1989).

[55] M. Wortis, M. Jarić, and U. Seifert, J. Molec. Liquids xx, xxx (1996). (in press)

[56] Where the prolate is the ground state, the ensemble is truly stationary. In regions of prolate metastability, there is an effectively stationary restricted ensemble, as long as the barrier height is appreciably larger than $k_{B} T$, as discussed in Sec. IIB.

[57] In principle, we can now calculate the corrections of order $k_{B} T / \omega$. In fact, we have not done so, because, in situations where these corrections are appreciable, there are other effects (non-Gaussian corrections, gravity) which are also important and harder to compute. For an improved level of comparison between theory and experiment, it will be crucial to take all these effects into account.

[58] This reversibility shows that the neck remains open in the budded state. The mechanism of the energy barrier to reverse budding has not been clearly identified. It may involve the intervention of short-range (e.g., van der Waals) forces across the narrow neck. "Denecking" seems always to be accompanied by some irreversible effects, i.e., one does not return precisely to the state of the original vesicle at a lower $T$.

[59] Vesicle A exhibited a small but detectable up-down asymmetry of unknown origin. This manifested itself in a nonzero value of $\left\langle a_{3}\right\rangle$, even in the prolate phase. For $v>$ 0.92 the observed value of $\left\langle a_{3}\right\rangle$ remained less than 0.005 . However, nearer the budding instability, we observed $\left\langle a_{3}\right\rangle \sim 0.04$, presumably because the high "susceptibility" near the transition magnifies some intrinsic asymmetry [25]. None of these effects are visible in a casual scan of the video images. One possible explanation is that vesicle A incorporated some structural "defect" at a scale below our resolution. Another possibility is that the gravitational "tipping" of the pear-fluctuations, which is discussed at the end of Sec. V, combined with too-short a sampling time to average out rare events produces spurious non-zero odd modes.

[60] D. Needham, T.J. McIntosh, and E. Evans, Biochem., 27, 4668 (1988).

[61] F.A. Nezil, and M. Bloom, Biophys. J. 61, 1176 (1992).

[62] Let the two sphere radii in suitable reduced units be $r_{1}$ and $r_{2}$ with $r=r_{1} / r_{2}$. The reduced area is $1=r_{1}^{2}+r_{2}^{2}$ and the reduced volume is $v=r_{1}^{3}+r_{2}^{3}$. Thus, we find $v=\left(1+r^{3}\right) /\left(1+r^{2}\right)^{3 / 2}$. 
[63] M. Kraus, PhD thesis, Universität Potsdam, 1996 (unpublished).

[64] These numbers reflect the relative densities of the interior and exterior solutions, as prepared. Small amounts of intermixing may have occurred during the course of the experiments, which would have the effect of decreasing $g$. We neglect the small variations in $g$ due to the temperature induced changes in $R_{A}$.

[65] K. A. Brakke, Experimental Mathematics 1, 141 (1992); for the code see the URL 'http://www.geom.umn.edu/software/evolver'.

[66] Thermal fluctuations tend to make the average vesicle outline more circular, so that we may expect that $\left\langle a_{2}\right\rangle$ and $\left\langle a_{4}\right\rangle$ go to zero when fluctuations become sufficiently strong. This effect would lead to a larger inferred value of $a_{4}^{(0)}$, which (via Fig. 2) would give a larger $\bar{c}_{0}$. 


\section{FIGURES}

FIG. 1. ADE phase diagram for $\alpha=1.4$, prolate region. First-order boundaries $(D)$ are indicated by solid lines; second-order boundaries $(C)$, by dashed lines; and, spinodals $(M)$, by dotted lines. Lowest-energy shapes are illustrated for each region. All symmetry axes are vertical as indicated for the prolate. Prolate shapes are locally stable between the upper spinodal line $M_{(0,-)}^{\text {pro }}$ and the lower spinodal line $M_{(2,+)}^{\text {pro }}$. The lines $D^{\text {pro/pear }}, C^{\text {pro/pear }}, D^{\text {pro/obl }}$, and $D^{\text {pro/nas }}$ bound the region where prolates are the lowest-energy shapes. In the region immediately above $D^{\text {pro/pear }}$ lowest-energy shapes are pear-like. $L^{\text {pear }}$, is the limiting line at which the neck of the pear shape shrinks to zero radius producing a vesiculated shape, as indicated. The lowest-energy states above $L^{\text {pear }}$ are dominated by vesiculated shapes. In the region immediately below the prolates, oblate and non-axisymmetric (nas) shapes have lowest energy [34]. The point $\mathrm{T}$ on the prolate/pear phase boundary is a tricritical point, separating first-order and second-order behavior. CEP labels a critical end point, where a second-order boundary $C^{\text {nas/obl }}$ (not shown, since it is very close to $D^{\text {pro/nas }}$ ) disappears underneath the lower prolate boundary. Note for future reference that the limiting line $L^{\text {pear }}$ crosses the upper spinodal line $M_{(0,-)}^{\text {pro }}$ of the prolate phase at $v=0.875$ for $\alpha=1.4$.

FIG. 2. The shape coefficient $a_{4}^{(0)}$ as a function of reduced volume $v$ for various values of the effective reduced spontaneous curvature $\bar{c}_{0}$, as indicated on the curves. These curves allow us to infer a value of $\bar{c}_{0}$, if $a_{4}^{(0)}$ is known at given $v$. This is the basis of the mapping procedure discussed in Sec. II . All the curves pass through $a_{4}^{(0)}=0$ at the sphere, $v=1$. Although $a_{4}^{(0)}$ is almost independent of $\bar{c}_{0}$ at $v=0.85$, there is no common crossing point.

FIG. 3. Time-sequence of phase-contrast video images of vesicle $\mathrm{A}$ at $v=0.954$. The images are ordered in time from the upper left to the lower right. The elapsed time between images is $6.3 \mathrm{~s}$. The length of the long vesicle axis is approximately $20 \mu \mathrm{m}$. The vesicle fluctuates about an axisymmetric prolate shape; however, each particular contour is different and, in general, non-axisymmetric.

FIG. 4. Time-sequence of phase-contrast video images of vesicle $\mathrm{A}$ at $v=0.912$. Times and scale are as in Fig. 3. The vesicle is now more elongated than it was in Fig. 3. Strong pear-like fluctuations in each direction are now clearly visible. 
FIG. 5. Time-sequence of phase-contrast video images of vesicle $\mathrm{A}\left(R_{A}=9.2 \mu \mathrm{m}\right)$ at $v \simeq 0.878$, illustrating the budding process. The scale is as in Fig. 3 and 4 . The time elapsed between images here is $1.2 \mathrm{~s}$. A pear-like fluctuation, much like those visible in Fig. \&, now carries the vesicle over the metastable barrier to the budded state. The pear shaped contours correspond to transient shapes and are not stable. Note that the ratio of the vesicle size to the satellite size after budding is roughly 2.8 .

FIG. 6. Time-sequence of phase-contrast video images of vesicle $\mathrm{B}\left(R_{A}=5.5 \mu \mathrm{m}\right)$ at $v \simeq 0.945$, illustrating the budding process for a smaller size vesicle. The time elapsed between images is the same as in Fig. 5. Here, the transition from the prolate via the transient pear to the budded state happens much more quickly than for vesicle $\mathrm{A}$, due to the smaller hydrodynamic radius.

FIG. 7. Typical time-series for the amplitudes $a_{3}$ and $a_{4}$ and for the effective reduced volume $v_{e}$ for vesicle $\mathrm{A}$ at reduced volume $\left\langle v_{e}\right\rangle=0.954$. The dashed line corresponds to the mean amplitude $\left\langle a_{n}\right\rangle$, which is close to zero for $a_{3}$ [59]. According to Eq. (22), the mean values $\left\langle v_{e}\right\rangle$ and $\left\langle a_{4}\right\rangle$ correspond to the zero-temperature quantities $v$ and $a_{4}^{(0)}$, respectively, which are the basis for the mapping.

FIG. 8. Same data as in Fig. 0 but for reduced volume $\left\langle v_{e}\right\rangle=0.912$. Note the longer time-scales for fluctuations in the $a_{3}$-mode as the shape instability (spinodal) is approached. 
FIG. 9. Experimental trajectories without (Sec. V, open symbols) and with (Sec. VI, filled symbols) gravitational corrections in the $\left(v, \bar{c}_{0}\right)$ diagram for vesicles $\mathrm{A}, \mathrm{B}$, and $\mathrm{C}$. Corrected and uncorrected data points are joined by a thin line. As explained in the text, the vesicle follows a path from lower right to upper left, as it is heated. Vesicles A and B underwent a budding instability after the upper-left-most point of the trajectory, vesicle $\mathrm{C}$ budded from the point shown. The spinodal lines $M^{\text {pro }}$ are the same as those shown in Fig. 1 and mark the upper and lower boundaries of the region predicted by theory to be locally stable for prolate shapes. Thus, for full consistency, the trajectories must terminate below the upper spinodal. Vesicles B and C satisfy this criterion. The final raw-data point on the trajectory of vesicle A is inconsistent with stability. Including gravitational effects moves the thermal trajectories to smaller reduced volume. For $g=2.2$ (appropriate only for vesicle A), the instability boundaries are shifted by gravity, as shown. The final point on the vesicle $A$ trajectory becomes consistent with theory, only when, in addition to gravitational effects on the mapping, the gravitational tipping of the fluctuating pear-like shapes is incorporated by excluding a cluster of data points (square symbols), as discussed at the end of Sec. \. Uncertainties are generally large near the spinodal lines and close to the sphere, where fluctuations become important.

FIG. 10. Experimental trajectories without (Sec. V, open symbols) and with (Sec. VI, filled symbols) gravitational corrections in the $\left(v, \bar{m}_{0}\right)$ phase diagram for vesicles $\mathrm{A}, \mathrm{B}$, and $\mathrm{C}$ using $\alpha=1.4$ (see Fig. 9 for a legend). The stability of data points does not depend on $\alpha$ and mirrors Fig. 9. As for Fig. 9, the last point of the vesicle A trajectory becomes consistent with the theoretically calculated stability when corrected for gravitational effects (including the cluster exclusion, as discussed in the text). A theoretical thermal trajectory (with the simple form $\bar{m}_{0} v=$ const) is shown for comparison. Although Figs. 9 and 10 look similar, they are not connected by a simple rescaling of the vertical axis.

FIG. 11. Computed gravitational corrections to the effective reduced volume $v_{e}$ at $g=2.2$, as is appropriate for vesicle A. The shift $\left(v_{e}-v\right)$ is shown vertically as a function of $\bar{c}_{0}$ and $v$. The dashed line corresponds to the prolate-oblate transition. All shifts meet this line with a slope different from -1 , as explained in the text. The shaded region $\left(v_{e}>1\right)$ is unphysical. 


\section{TABLES}

\begin{tabular}{|c|c|c|c|}
\hline Vesicle & $T / \mathrm{C}^{\circ}$ & $\overline{\left\langle v_{e}\right\rangle}$ & $\overline{\left\langle a_{4}\right\rangle}$ \\
\hline \multirow{5}{*}{ A } & $28.7 \pm 0.01$ & $0.954 \pm 0.001$ & $-0.0037 \pm 0.0011$ \\
\hline & $\overline{32.7 \pm 0.01}$ & $0.932 \pm 0.001$ & $0.0054 \pm 0.0013$ \\
\hline & $\overline{37.8 \pm 0.01}$ & $0.912 \pm 0.001$ & $0.0038 \pm 0.0022$ \\
\hline & $42.4 \pm 0.01$ & $0.894 \pm 0.001$ & $0.0092 \pm 0.001$ \\
\hline & $\overline{45.8 \pm 0.01 \text { budding }}$ & $0.878 \pm 0.002$ & $\mathrm{~N} / \mathrm{A}$ \\
\hline \multirow{3}{*}{ B } & $32.9 \pm 0.01$ & $0.970 \pm 0.001$ & $0.005 \pm 0.001$ \\
\hline & $40.3 \pm 0.01$ & $0.950 \pm 0.001$ & $0.016 \pm 0.001$ \\
\hline & $\overline{42.0 \pm 0.01 \text { budding }}$ & $0.945 \pm 0.002$ & $\mathrm{~N} / \mathrm{A}$ \\
\hline $\mathrm{C}$ & $27.0 \pm 0.01$ budding & $0.983 \pm 0.001$ & $0.008 \pm 0.002$ \\
\hline
\end{tabular}

TABLE I. Experimental results for vesicles $A, B$, and $C$. At each temperature $T$, the values of $\left\langle v_{e}\right\rangle$, and $\left\langle a_{4}\right\rangle$ are shown. The final budding temperature is also given, along with the (for $\mathrm{A}$ and $\mathrm{B}$ extrapolated) reduced volume at budding. 


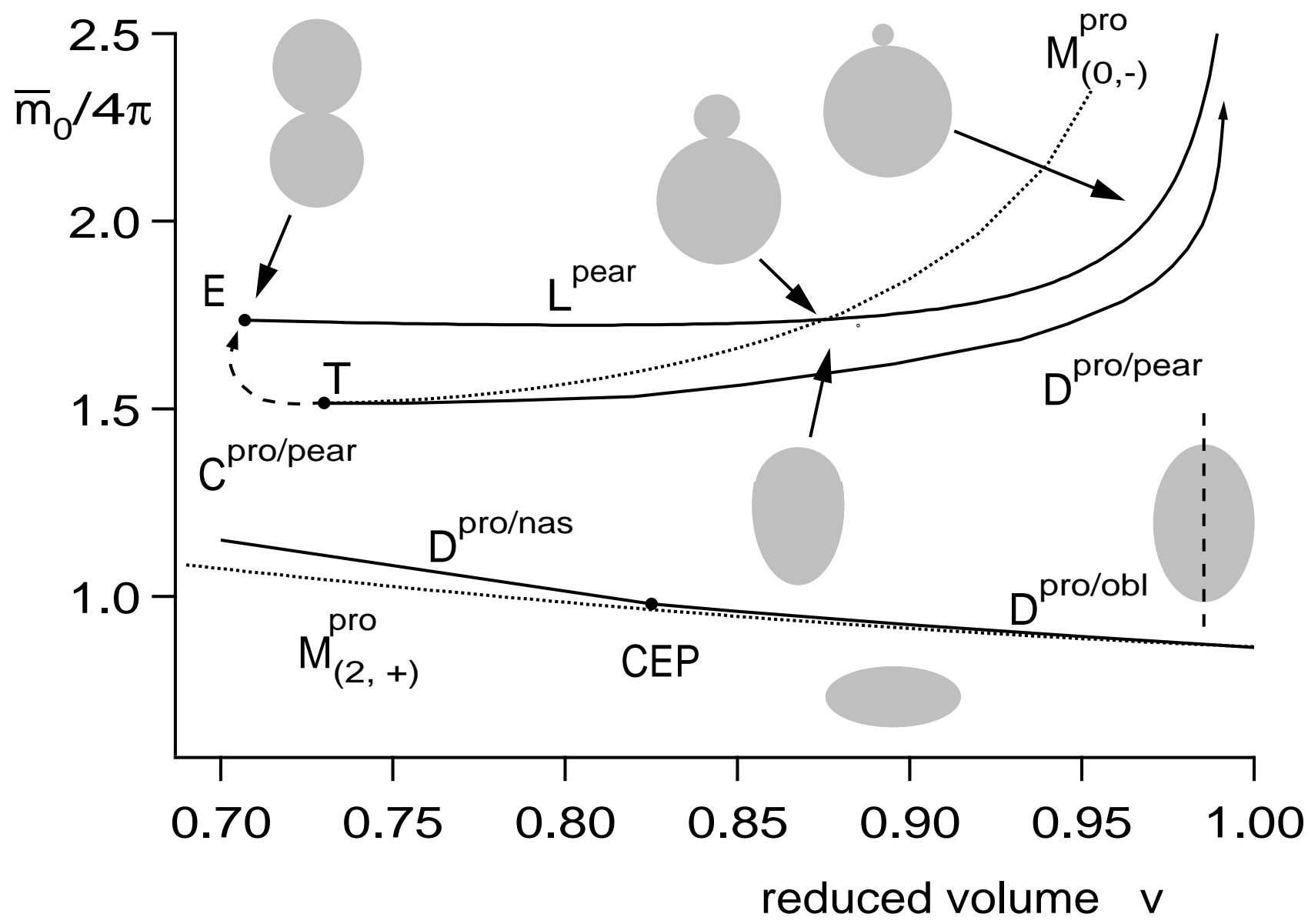

Fig. 1: Döbereiner et al. 


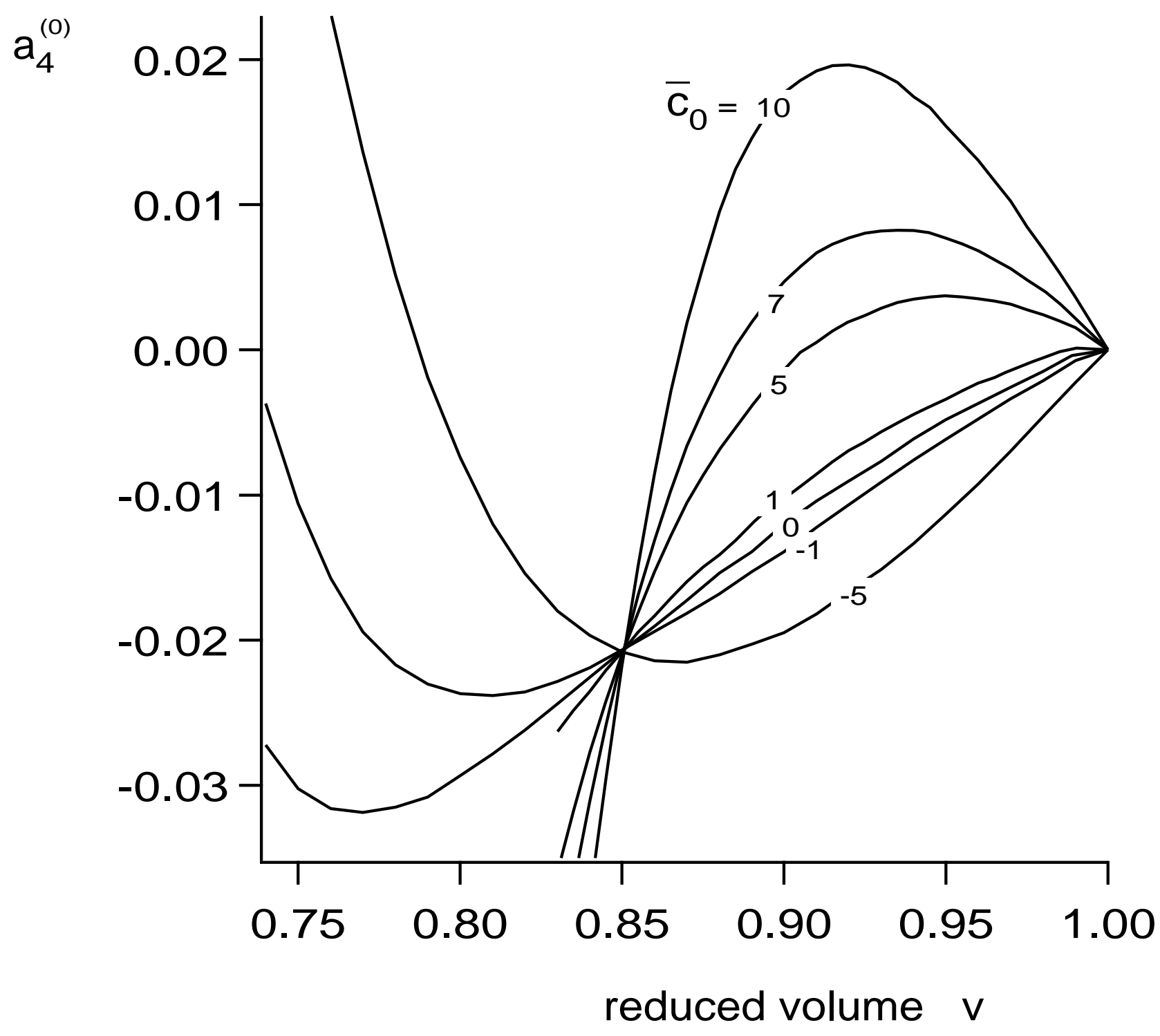

Fig. 2: Döbereiner et al. 


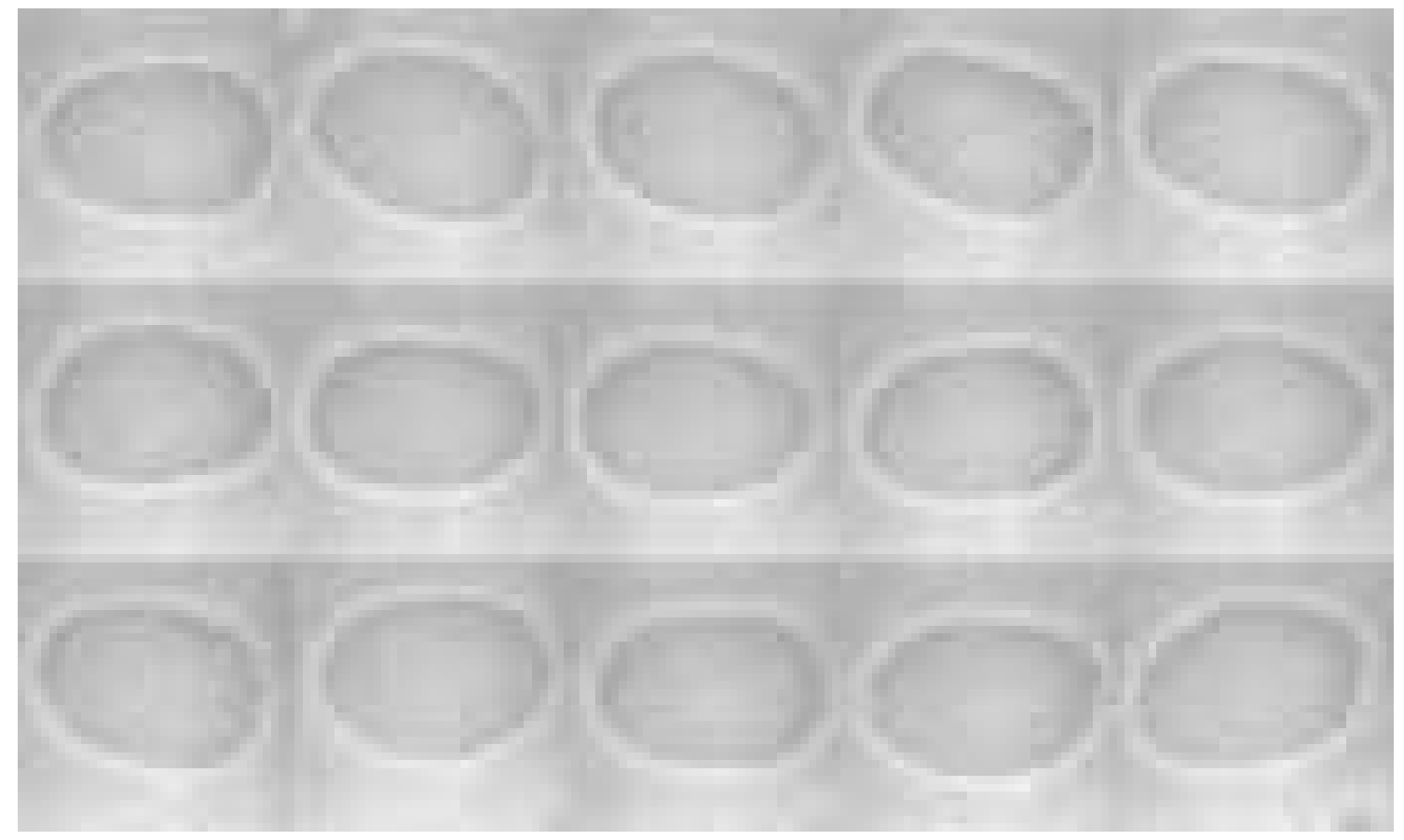

Fig. 3: Döbereiner et al. 


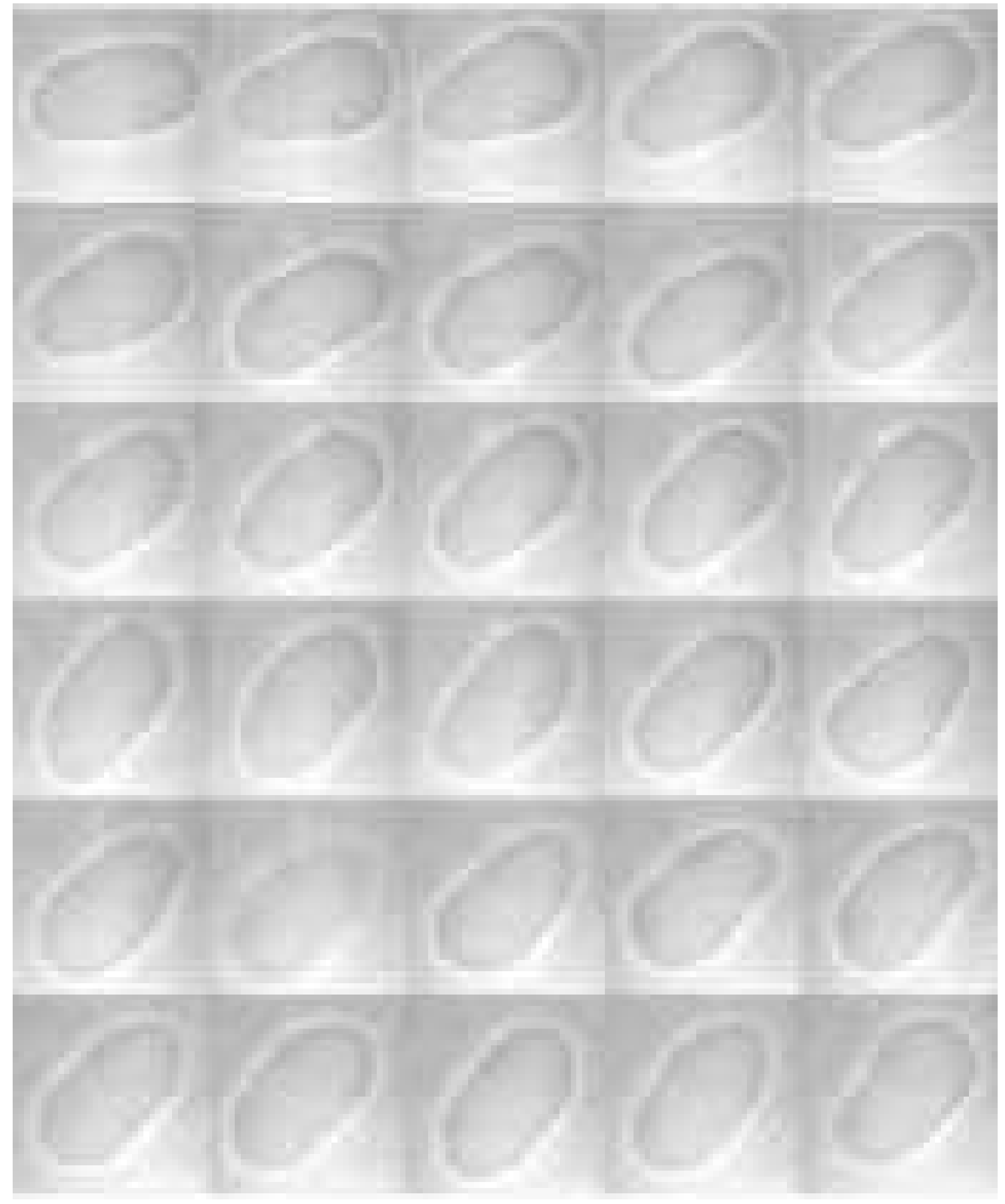

Fig. 4: Döbereiner et al. 
Fig. 5: Döbereiner et al. 


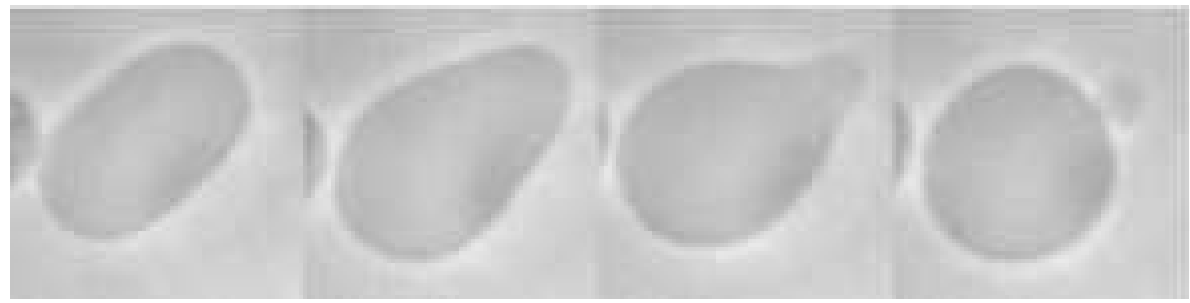

Fig. 6: Döbereiner et al. 

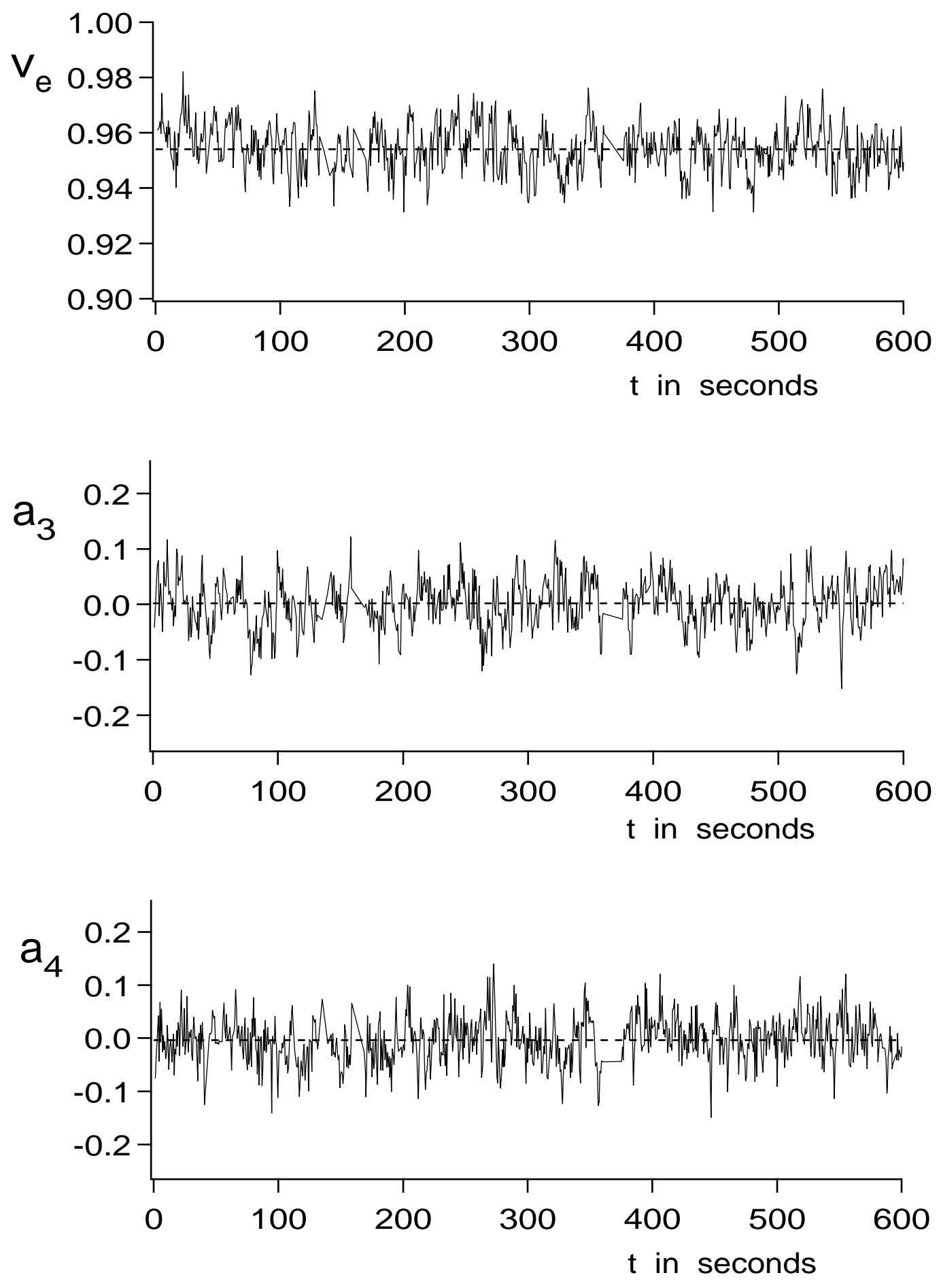

Fig. 7: Döbereiner et al. 

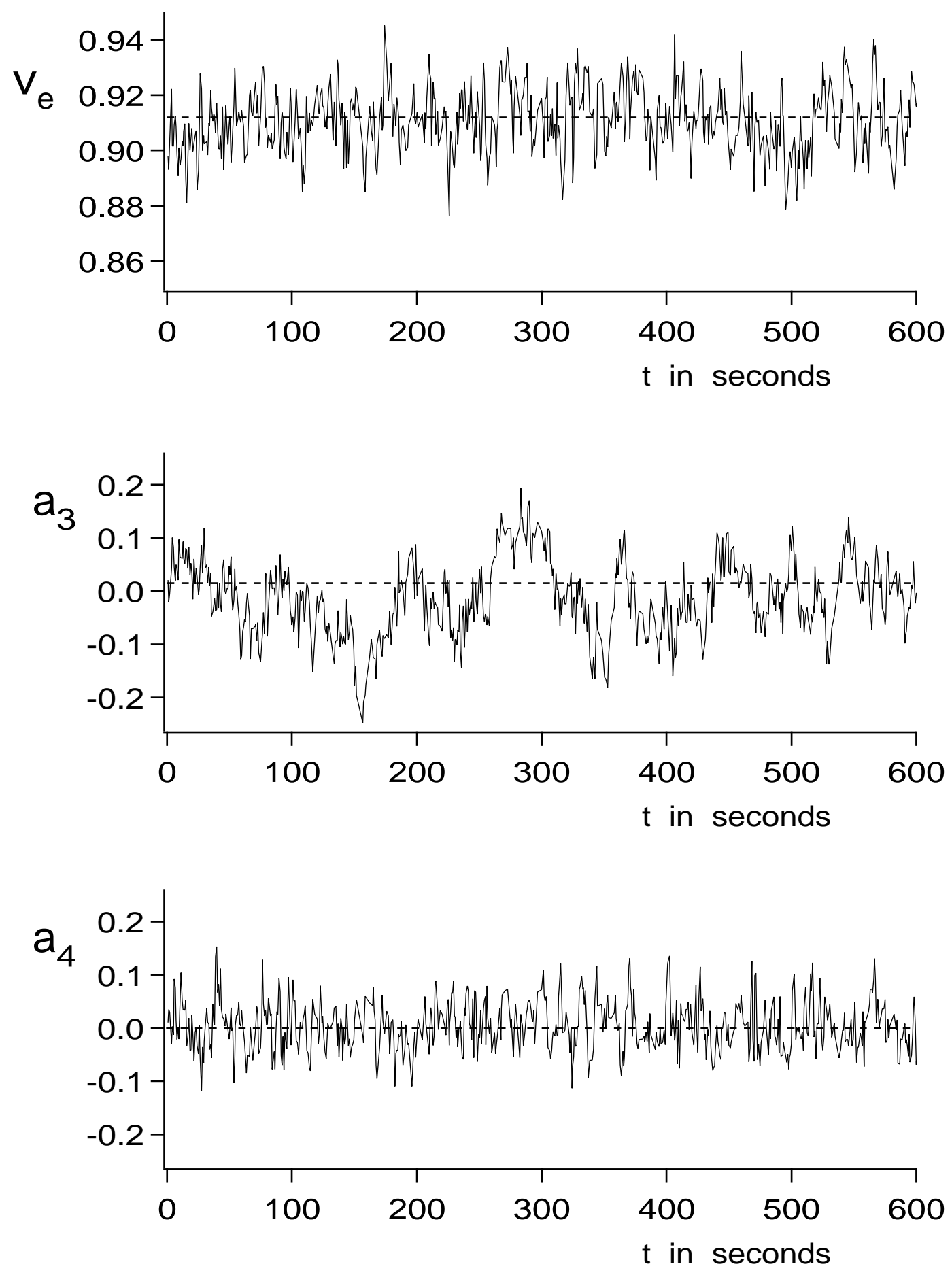

Fig. 8: Döbereiner et al. 


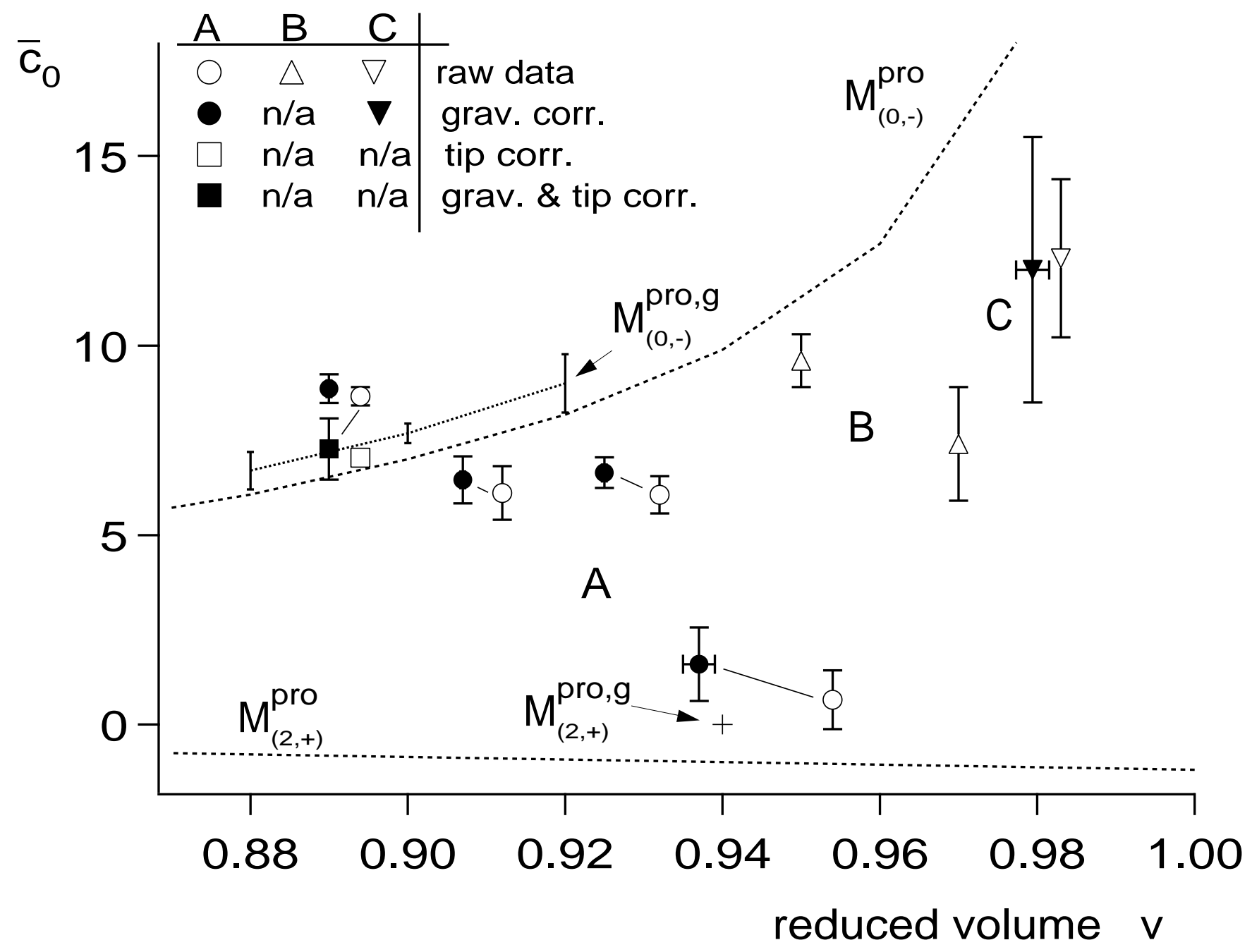

Fig. 9: Döbereiner et al. 


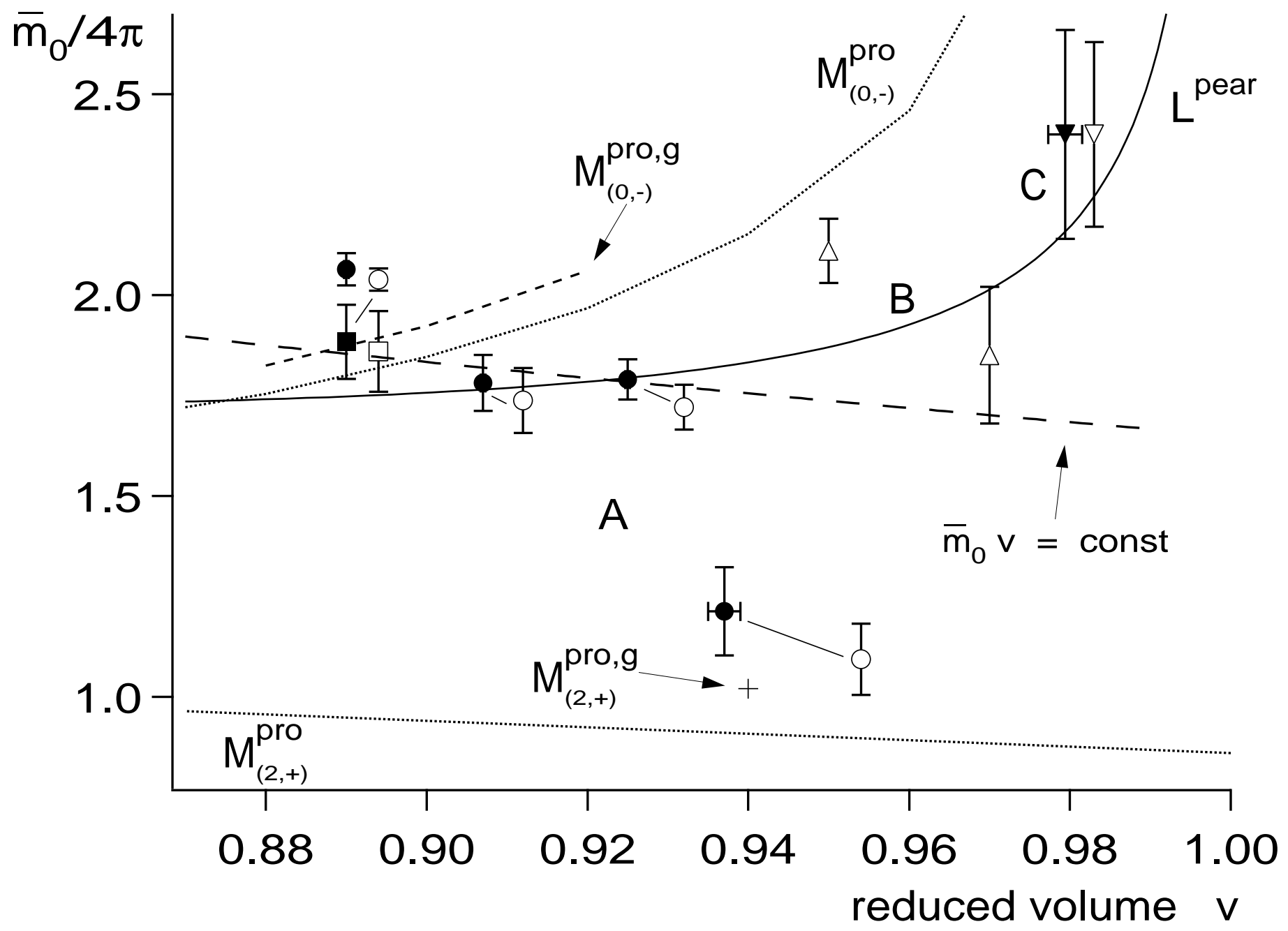

Fig. 10: Döbereiner et al. 


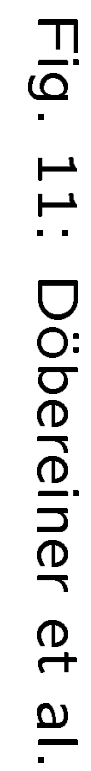

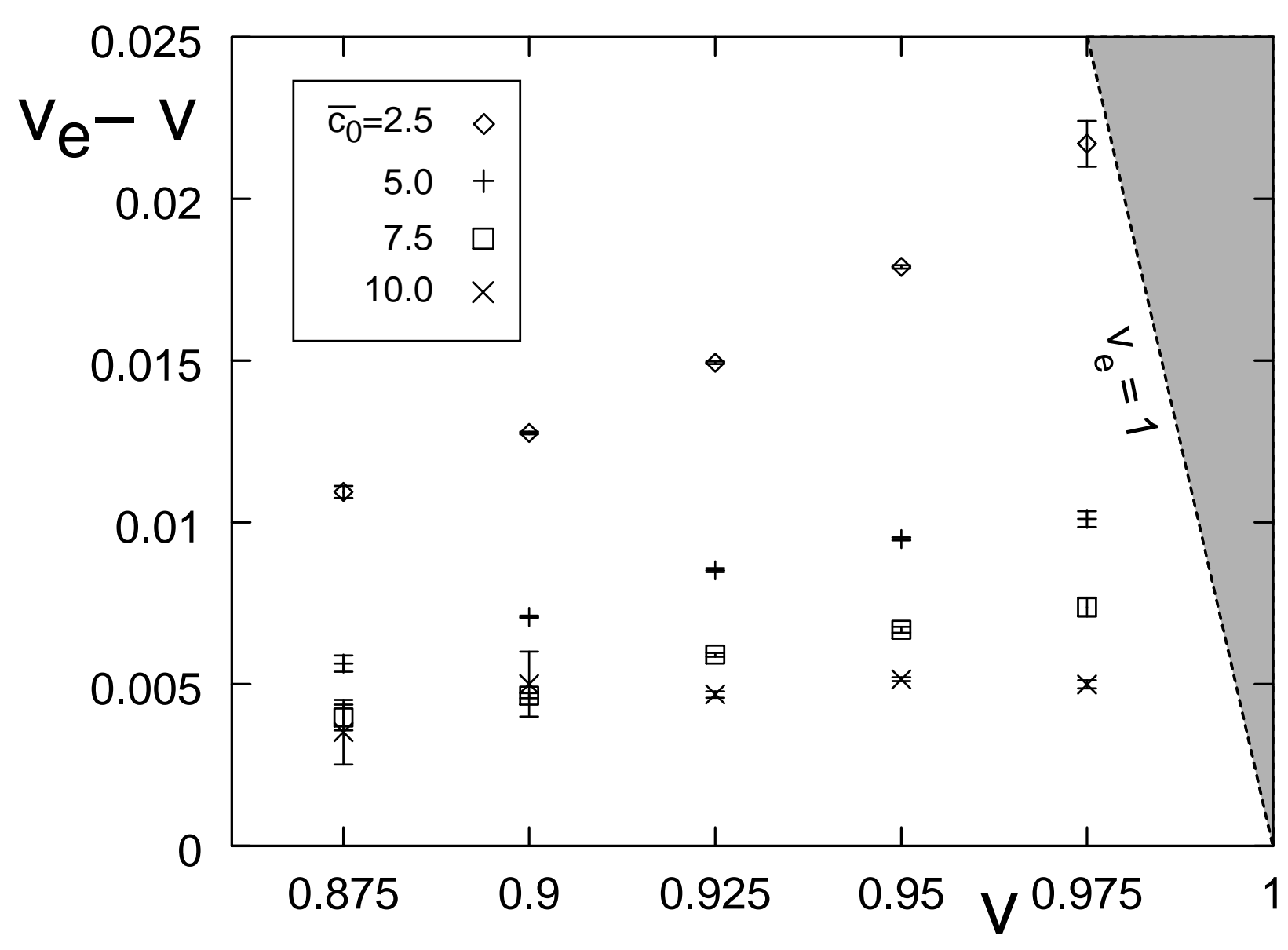

